\title{
JONES POLYNOMIALS OF ALTERNATING LINKS
}

\author{
KUNIO MURASUGI
}

\begin{abstract}
Let $J_{K}(t)=a_{r} t^{r}+\cdots+a_{s} t^{s}, r>s$, be the Jones polynomial of a knot $K$ in $S^{3}$. For an alternating knot, it is proved that $r-s$ is bounded by the number of double points in any alternating projection of $K$. This upper bound is attained by many alternating knots, including 2-bridge knots, and therefore, for these knots, $r-s$ gives the minimum number of double points among all alternating projections of $K$. If $K$ is a special alternating knot, it is also proved that $a_{s}=1$ and $s$ is equal to the genus of $K$. Similar results hold for links.
\end{abstract}

1. Introduction. Let $K$ be an oriented knot or link in $S^{3}$ and let $J_{K}(t)$ be the polynomial defined by V. Jones [5] which is now called the Jones polynomial of $K$. $J_{K}(t)$ is an invariant of a knot or link type. It is not clear, however, to what extent $J_{K}(t)$ is related to known algebraic or topological invariants in knot theory. Although it is shown [10] that $J_{K}(t)$ determines the Arf invariant of a knot or a link, it is also known that $J_{K}(t)$ does not determine the genus or the signature of $K[8]$.

In this paper, we prove that for an alternating knot, $J_{K}(t)$ provides some information that has never been obtained from other algebraic invariants. To be more precise, let $K$ be an alternating knot in $S^{3}$ and $\tilde{K}$ an alternating projection of $K$. Let $J_{K}(t)$ be the Jones polynomial of $K{ }^{1}{ }^{1}$ Write $J_{K}(t)=a_{r} t^{r}+\cdots+a_{s} t^{s}$, where $r>s$ and $a_{r} \neq 0 \neq a_{s}$. Let $h(\tilde{K})$ be the number of double points in $\tilde{K}$. Then we can prove

THEOREM A. $r-s \leq h(\tilde{K})$.

It is probably not surprising that the "reduced" degree $r-s$ is bounded by the number of double points of $\tilde{K}$, but what is surprising is the fact that equality in Theorem A holds for many alternating knots including alternating algebraic knots [Theorem 11.2], alternating pretzel knots and alternating closed 3-braids $\beta=\sigma_{1}^{p_{1}} \sigma_{2}^{-q_{1}} \cdots \sigma_{1}^{p_{r}} \sigma_{2}^{-q_{r}}$, where $r \geq 2$ and $p_{i}, q_{i}>0$ [5]. It means that for these knots, $h(\tilde{K})$ is a knot type invariant and $h(\tilde{K})$ gives the minimum number of double points among all alternating projections of $K$. This proves

THEOREM B (SEE COROLLARY 9.3). Let $K$ be an alternating knot and $\tilde{K}$ an alternating projection. If $r-s=h(\tilde{K})$, then for any alternating projection $K^{*}$ of $K$, the number of double points of $K^{*}$ is at least $h(\tilde{K})$. Therefore, $\tilde{K}$ is an

Received by the editors April 9, 1985 and, in revised form, May 25, 1985.

1980 Mathematics Subject Classification. Primary 57M25.

Key words and phrases. Knot, link, Jones polynomial, alternating knot, reduced Alexander polynomial.

${ }^{1}$ The original Jones polynomial $V_{K}(t)$ in [5] is given by $J_{K}\left(t^{-1}\right)$

(C)1986 American Mathematical Society $0002-9947 / 86 \$ 1.00+\$ .25$ per page 
alternating projection of $K$ with a minimum number of double points. ( $\tilde{K}$ will be called a minimum alternating projection.)

Using Theorem $\mathrm{B}$, for instance, we are able to determine the minimum number of double points an alternating projection of a 2-bridge knot can have (see Corollary 11.3).

Until the present the minimum number of double points of a knot projection has been determined only for very limited knot types $[\mathbf{1}, \mathbf{3}]$.

Since it is unlikely that a nonalternating projection of an alternating knot has fewer double points, it is plausible that the minimum number of double points of an alternating knot will be given by $r-s$.

Now, since an alternating knot is "formed" from special alternating knots or links, a proof of Theorem A requires more information on Jones polynomials on special alternating knots. Let $K$ be a special alternating knot in $S^{3}$. That is, $K$ has a connected alternating projection in which one of the chessboard surfaces is orientable. Let $\tilde{K}$ be such a special alternating projection. For simplicity, we assume that at each double point of $\tilde{K}$, an oriented segment crosses over the other from left to right

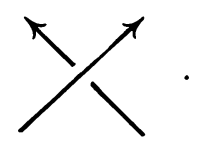

We say that $\tilde{K}$ is of positive type. (Otherwise, $\tilde{K}$ is of negative type.) A special alternating knot has a special alternating projection of either positive or negative type, but it cannot have both types. For these knots, we can prove slightly more precise results.

THEOREM C (SEE THEOREM 2.1). Let $K$ be a special alternating knot which has a special alternating projection $\tilde{K}$ of positive type. Let $J_{K}(t)$ be the Jones polynomial of $K$. Write $J_{K}(t)=a_{r} t^{r}+\cdots+a_{s} t^{s}$, where $r>s$ and $a_{r} \neq 0 \neq a_{s}$. Then

(I) $a_{s}=1$.

(II) $s \geq 0$ and $s$ is equal to the genus of $K$.

(III) Let $h(\tilde{K})$ be the number of double points in $\tilde{K}$. Then

$$
r-s \leq h(\tilde{K}) .
$$

Note that if $K$ is of negative type, then $K$ is the mirror image of a special alternating knot $K^{*}$ of positive type, and, hence, $J_{K}(t)=J_{K^{*}}\left(t^{-1}\right)$, and the rôles of $r$ and $s$ should be reversed.

Theorem A, in principal, follows from Theorem C. It should be noted here that Theorems A and C are, in fact, proved for alternative links introduced by L. H. Kauffman [7].

One of the outstanding problems still unsolved is whether any alternating projection of an alternating link without removable points

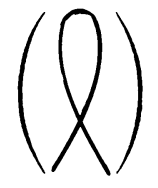


is minimum. The answer is not known even for 2-bridge links, for which we now have the minimum alternating projection.

This paper is organized as follows. In $\S 2$, we state the first main theorem (Theorem 2.1) that is a slight generalization of Theorem $C$. The proof of this theorem will be completed at the end of $\S 8$. In $\S \S 9-10$, we will prove the second main theorem (Theorem 9.1) that implies Theorem A. In $\S 11$, we will prove that equality in Theorem A holds for alternating algebraic links considered in [13].

2. Main Theorem I. In order to prove Theorem C, we need to consider links rather than knots, and to this purpose, the polynomials defined in [8] will be more convenient than the original Jones polynomials.

Let $L$ be an oriented link in $S^{3}$. Let $P_{L}(l, m)$ be the polynomial in two variables $l$ and $m$ introduced in [8]. $P_{L}(l, m)$ is defined recursively by using the following fundamental identities $(2.1)^{2}$ and (2.2).

(I) If $L_{+}, L_{-}$and $L_{0}$ are completely identical projections except at one double point $c$, where they are related by Figure 1, then

$$
l P_{L_{-}}(l, m)+l^{-1} P_{L_{+}}(l, m)+m P_{L_{0}}(l, m)=0 .
$$

(II) If $L$ is a trivial link of $n$ components, then

$$
P_{L}(l, m)=\left(-\left(l+l^{-1}\right) / m\right)^{n-1}
$$

Now for an $n$-component link $L$ in $S^{3}$, we define the polynomial $Q_{L}(l, m)$ by

$$
Q_{L}(l, m)=(m / l)^{n-1} P_{L}(l, m)
$$

$Q_{L}(l, m)$ is an integer polynomial in $l^{ \pm 2}$ and $m^{2}$; i.e.,

$$
Q_{L}(l, m) \in \mathbf{Z}\left[m^{2}, l^{2}, l^{-2}\right]
$$
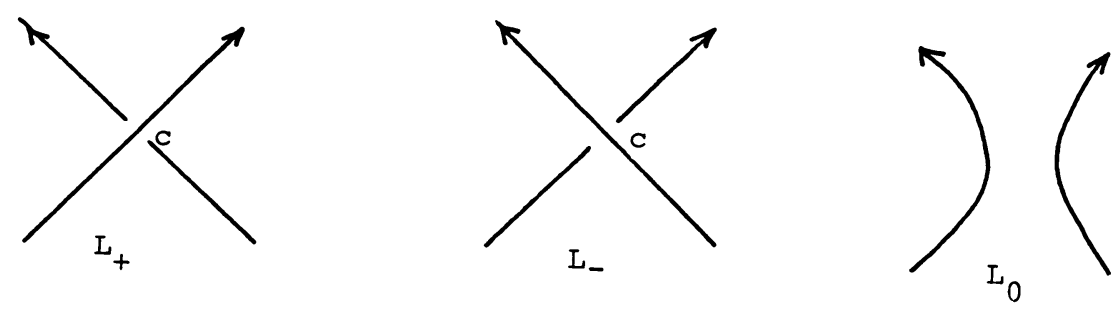

FIGURE 1

\footnotetext{
${ }^{2}$ Formula (2.1) is slightly different from the original formula given in $[8] . P_{L_{+}}(l, m)$ is actually $K_{L_{+}}\left(l^{-1}, m\right)$ in $[8]$.
} 

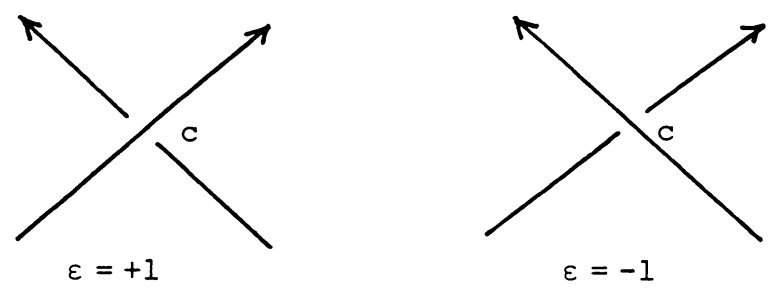

FIGURE 2

Finally we define $J_{L}(t)$ as

$$
J_{L}(t)=Q_{L}(t i,-(\sqrt{t}-1 / \sqrt{t}) i)
$$

where $i^{2}=-1$.

$J_{L}(t)$ is an integer polynomial in $t^{ \pm 1}$. If $K$ is a knot, then $J_{K}\left(t^{-1}\right)$ coincides with the original Jones polynomial. However, if $L$ is a link of $n(>1)$ components, $J_{L}(t)$ will be called the reduced Jones polynomial.

EXAMPLE 2.1. For an $n$-component trivial link $L$,

$$
\begin{aligned}
Q_{L}(l, m) & =\left(\frac{m}{l}\right)^{n-1} P_{L}(l, m)=\left(\frac{m}{l}\right)^{n-1}\left(-\frac{l+l^{-1}}{m}\right)^{n-1} \\
& =(-1)^{n-1}\left(1+l^{-2}\right)^{n-1}
\end{aligned}
$$

and, hence, $J_{L}(t)=(-1)^{n-1}\left(1-t^{-2}\right)^{n-1}$.

Let $\tilde{L}$ be a link diagram of a link $L$; i.e., $\tilde{L}$ is a projection of $L$ on $S^{2}$. To each double point $c$ of $\tilde{L}$, we assign $\varepsilon(c)=+1$ or -1 as shown in Figure 2 . $\tilde{L}$ is said to have positive (negative) type if $\varepsilon(c)=+1(\varepsilon(c)=-1)$ for all double points $c$ in $\tilde{L}$. An oriented link $L$ is called a link of positive (negative) type, or simply a positive (negative) link if $L$ has a diagram of positive (negative) type. A special alternating link is a link that has an alternating and positive (negative) link diagram.

The family of positive or negative (not necessarily alternating) links includes positive or negative closed braids; in particular, it includes any torus links.

The main theorem I (Theorem 2.1) of this paper holds not only for special alternating links but also for positive or negative links.

THEOREM 2.1. Let $L$ be an oriented $n$-component positive link. Let $\tilde{L}$ be a link diagram. Suppose that $\tilde{L}$ consists of $\lambda$ connected components $\tilde{L}_{1}, \tilde{L}_{2}, \ldots, \tilde{L}_{\lambda}$, and that each component $\tilde{L}_{i}$ is of positive type. Let $L_{i}$ be the sublink of $L$ whose diagram is $\tilde{L}_{i} .\left(L_{i}\right.$ is a positive link.) Let $g\left(L_{i}\right)$ denote the genus of $L_{i}$, and $h\left(\tilde{L}_{i}\right)$ the number of double points on $\tilde{L}_{i}$. Write $J_{L}(t)=a_{r} t^{r}+\cdots+a_{s} t^{s}$, where $r>s$ and $a_{r} \neq 0 \neq a_{s}$. Then

$$
\begin{aligned}
& \text { (1) } a_{s}=1 \\
& \text { (2) } s=\sum_{i=1}^{\lambda} g\left(L_{i}\right)-(n-1)-(\lambda-1), \\
& \text { (3) } r-s \leq \sum_{i=1}^{\lambda} h\left(\tilde{L}_{i}\right)+(n-1)+(\lambda-1) .
\end{aligned}
$$


Note that for a general positive link $L$, equality in (3) may not hold. The simplest example is the closure $\hat{\beta}$ of a 3 -braid $\beta=\left(\sigma_{1} \sigma_{2}\right)^{2} . \hat{\beta}$ is a trefoil knot, but the natural diagram of the closed braid contains four double points.

COROLlaRY 2.2. In Theorem 2.1, if

$$
r-s=h(\tilde{L})+(n-1)+(\lambda-1),
$$

then for any positive diagram of $L$, the number of double points is at least $h(\tilde{L})$. Therefore, $\tilde{L}$ is a minimum positive diagram of $L$.

Since $h(\tilde{L}), n$ and $\lambda$ are independent from the orientation, if equality holds in (2.5)(3), Corollary 2.2 yields a curious conclusion that $s$ depends on the orientation of $L$ but $r-s$ does not.

3. Proof of Theorem 2.1 (I). First we fix notation that will be used throughout this paper. All links are oriented.

Given a link $L$, a diagram of $L$ is usually denoted by $\tilde{L}$, and from the context, it is generally easy to understand which diagram is being referred to.

For a link $L$ :

(1) $\lambda(L)$ is the number of sublinks of $L$ which split each other ,

(2) $\mu(L)$ is the number of components of $L$,

(3) $\delta(L)$ is the degree of the reduced Alexander polynomial of $L$.

For a link diagram $\tilde{L}$,

(4) $\lambda(\tilde{L})$ is the number of connected components of $\tilde{L}$,

(5) $h(\tilde{L})$ is the number of double points on $\tilde{L}$.

For a Laurent polynomial $f(t)=\sum_{j=s}^{r} a_{j} t^{j}, a_{r} \neq 0 \neq a_{s}, r>s$,

(6) $\quad l-\operatorname{deg} f(t)=s, \quad h-\operatorname{deg} f(t)=r, \quad r-\operatorname{deg} f(t)=r-s$, $l$-coef $f(t)=a_{s}, \quad h$-coef $f(t)=a_{r}$.

It is well known $[\mathbf{2 , 1 1}]$ that if $\tilde{L}$ is a positive (negative) diagram of $L$, then $\lambda(\tilde{L})=\lambda(L)$. In other words, a positive (negative) link $L$ is not split iff its diagram is connected.

Since a diagram $\tilde{L}$ uniquely determines a link $L$, we may write $\mu(\tilde{L})$ or $\delta(\tilde{L})$ for $\mu(L)$ or $\delta(L)$. To emphasize a link $L$, we frequently write $P(L ; l, m)$ or $Q(L ; l, m)$ for $P_{L}(l, m)$ or $Q_{L}(l, m)$.

Now let $L, L_{i}, \tilde{L}, \tilde{L}_{i}$ and $J_{L}(t)$ be defined as in Theorem 2.1. Let $J_{i}(t)$ denote the reduced Jones polynomial of $L_{i}$ defined by (2.4). First we prove

PROPOSITION 3.1. Let $\lambda=\lambda(L)$.

(1) If $l$-coef $J_{i}(t)=1,1 \leq i \leq \lambda$, then $l$-coef $J(t)=1$.

(2) $l-\operatorname{deg} J(t)=\sum_{i=1}^{\lambda} l-\operatorname{deg} J_{i}(t)-2(\lambda-1)$.

(3) $h-\operatorname{deg} J(t)=\sum_{i=1}^{\lambda} h-\operatorname{deg} J_{i}(t)$. 
PROOF. Since $L$ consists of exactly $\lambda$ split sublinks $L_{1}, L_{2}, \ldots, L_{\lambda}$, it follows from $[8,(3)]$ that

$$
P(L ; l, m)=(-1)^{\lambda-1}\left(\frac{l+l^{-1}}{m}\right)^{\lambda-1} \prod_{i=1}^{\lambda} P\left(L_{i} ; l, m\right) .
$$

Since

$$
n-1=\sum_{i=1}^{\lambda} \mu\left(L_{i}\right)-1=\sum_{i=1}^{\lambda}\left\{\mu\left(L_{i}\right)-1\right\}+(\lambda-1),
$$

it follows from (3.2) that

$$
\begin{aligned}
Q(L ; l, m) & =\left(\frac{m}{l}\right)^{n-1} P(L ; l, m) \\
& =(-1)^{\lambda-1}\left(\frac{m}{l}\right)^{n-1}\left(\frac{l+l^{-1}}{m}\right)^{\lambda-1} \prod_{i=1}^{\lambda} P\left(L_{i} ; l, m\right) \\
& =(-1)^{\lambda-1}\left(\frac{m}{l}\right)^{\lambda-1}\left(\frac{l+l^{-1}}{m}\right)^{\lambda-1} \prod_{i=1}^{\lambda} Q\left(L_{i} ; l, m\right) \\
& =(-1)^{\lambda-1}\left(1+l^{-2}\right)^{\lambda-1} \prod_{i=1}^{\lambda} Q\left(L_{i} ; l, m\right) .
\end{aligned}
$$

Therefore, we have

$$
J_{L}(t)=(-1)^{\lambda-1}\left(1-t^{-2}\right)^{\lambda-1} \prod_{i=1}^{\lambda} J_{i}(t)
$$

from which Proposition 3.1 follows immediately.

Now the proof of Theorem 2.1 will be by induction on $h(\tilde{L})=\sum_{i=1}^{\lambda} h\left(\tilde{L}_{i}\right)$, the total number of double points on $\tilde{L}$.

As we have seen in Example 2.1, Theorem 2.1 is true for $h(\tilde{L})=0$. (Furthermore, $r-\operatorname{deg} J_{L}(t)=(n-1)+(\lambda-1)$.) Therefore, we may assume that Theorem 2.1 holds for a positive link $L^{*}$ with $h\left(\tilde{L}^{*}\right)<h(\tilde{L})$.

First, we will show that it is sufficient to prove Theorem 2.1 for a nonsplit positive link.

Assume that Theorem 2.1 is proved for $\lambda=1$. Suppose $\lambda>1$. Then two cases can occur.

Case 1. $h\left(\tilde{L}_{i}\right)<h(\tilde{L})$ for all $i=1,2, \ldots, \lambda$.

Case 2. $h\left(\tilde{L}_{i}\right)=h(\tilde{L})$ for some $i$, and $h\left(\tilde{L}_{j}\right)=0$ for $j \neq i$, and hence $\tilde{L}_{j}$ is a circle on $S^{2}$.

For Case 1, we apply the induction hypothesis on each $L_{i}$, and we have

$$
l-\operatorname{deg} J_{i}(t)=g\left(L_{i}\right)-\left\{\mu\left(L_{i}\right)-1\right\}
$$

and

$$
r-\operatorname{deg} J_{i}(t) \leq h\left(\tilde{L}_{i}\right)+\left\{\mu\left(L_{i}\right)-1\right\}
$$


Therefore, Proposition 3.1 and the induction hypothesis imply that

(1) $l$-coef $J_{L}(t)=1$,

(2) $l-\operatorname{deg} J_{L}(t)=\sum_{i=1}^{\lambda} l-\operatorname{deg} J_{i}(t)-2(\lambda-1)$

$$
\begin{aligned}
& =\sum_{i=1}^{\lambda}\left\{g\left(L_{i}\right)-\mu\left(L_{i}\right)+1\right\}-2(\lambda-1) \\
& =\sum_{i=1}^{\lambda} g\left(L_{i}\right)-\sum_{i=1}^{\lambda} \mu\left(L_{i}\right)+\lambda-2(\lambda-1) \\
& =\sum_{i=1}^{\lambda} g\left(L_{i}\right)-(n-1)-(\lambda-1)
\end{aligned}
$$

(3) $\quad r-\operatorname{deg} J_{L}(t)=\sum_{i=1}^{\lambda} h-\operatorname{deg} J_{i}(t)-\sum_{i=1}^{\lambda} l-\operatorname{deg} J_{i}(t)+2(\lambda-1)$

$$
\begin{aligned}
& =\sum_{i=1}^{\lambda} r-\operatorname{deg} J_{i}(t)+2(\lambda-1) \\
& \leq \sum_{i=1}^{\lambda}\left\{h\left(\tilde{L}_{i}\right)+\mu\left(L_{i}\right)-1\right\}+2(\lambda-1) \\
& =\sum_{i=1}^{\lambda} h\left(\tilde{L}_{i}\right)+n-\lambda+2(\lambda-1) \\
& =\sum_{i=1}^{\lambda} h\left(\tilde{L}_{i}\right)+(n-1)+(\lambda-1) .
\end{aligned}
$$

If Case 2 occurs, then $L$ splits into one link $L_{i}$ and a trivial $(\lambda-1)$-component link, and hence,

$$
P(L ; l, m)=P\left(L_{i} ; l, m\right)\left(-\left(l+l^{-1}\right) / m\right)^{\lambda-1} .
$$

Therefore, we have

$$
Q(L ; l, m)=\left(\frac{m}{l}\right)^{n-1} P\left(L_{i} ; l, m\right)(-1)^{\lambda-1}\left(\frac{l+l^{-1}}{m}\right)^{\lambda-1} .
$$

Since $n=\mu_{i}+\lambda-1, \mu_{i}=\mu\left(L_{i}\right)$, we see from (3.4) that

$$
\begin{aligned}
Q(L ; l, m) & =(m / l)^{\mu_{i}-1} P\left(L_{i} ; l, m\right)(-1)^{\lambda-1}\left(1+l^{-2}\right)^{\lambda-1} \\
& =Q\left(L_{i} ; l, m\right)(-1)^{\lambda-1}\left(1+l^{-2}\right)^{\lambda-1},
\end{aligned}
$$

and hence

$$
J_{L}(t)=(-1)^{\lambda-1}\left(1-t^{-2}\right)^{\lambda-1} J_{i}(t) .
$$

Since $l-\operatorname{deg} J_{i}(t)=g\left(L_{i}\right)-\left(\mu_{i}-1\right)$, it follows from Proposition 3.1(2) that

$$
\begin{aligned}
l-\operatorname{deg} J_{L}(t) & =g\left(L_{i}\right)-\left(\mu_{i}-1\right)-2(\lambda-1) \\
& =g\left(L_{i}\right)-\left(\mu_{i}+\lambda-2\right)-(\lambda-1)=g\left(L_{i}\right)-(n-1)-(\lambda-1) .
\end{aligned}
$$


Furthermore, since $h$-deg $J_{L}(t)=h$-deg $J_{i}(t)$, it follows from Proposition 3.1(2), (3) that

$$
\begin{aligned}
r-\operatorname{deg} J_{L}(t) & =h-\operatorname{deg} J_{L_{i}}(t)-l-\operatorname{deg} J_{L_{i}}(t)+2(\lambda-1) \\
& =r-\operatorname{deg} J_{L_{i}}(t)+2(\lambda-1) \leq h\left(\tilde{L}_{i}\right)+\left(\mu_{i}-1\right)+2(\lambda-1) \\
& =h\left(\tilde{L}_{i}\right)+\left(\mu_{i}+\lambda-2\right)+(\lambda-1)=h(\tilde{L})+(n-1)+(\lambda-1) .
\end{aligned}
$$

This proves Theorem 2.1. Therefore, in the following proof, we may assume that $L$ is not split.

4. Proof of Theorem 2.1 (II). Let $L$ be a nonsplit positive link and $\tilde{L}$ its positive diagram. To compute $P(L ; l, m)$, we apply the fundamental identities at several double points on $\tilde{L}$ to make $\tilde{L}$ "simpler".

Let $L_{+}, L_{-}$and $L_{0}$ be link diagrams described in $\S 2(\mathrm{I})$. For convenience, we say that $L_{-}\left(\right.$or $\left.L_{+}\right)$is obtained from $L_{+}$(or $L_{-}$) by changing the incident sign $\varepsilon$ at $c$, and $L_{0}$ is obtained from $L_{-}$(or $L_{+}$) by eliminating the double point $c$.

Now we study the effect of an elimination of a double point $c$ from a link diagram.

First we recall a relation between $g(L)$ and $\delta(L)$ for a positive (negative) link $K$. Let $\tilde{L}$ be a connected positive diagram. $\tilde{L}$ divides $S^{2}$ into finitely many domains.

It is known that $\tilde{L}$, considered as a 1-complex in $S^{2}$, is divided into finitely many oriented circles in $S^{2}$, called Seifert circles in $[\mathbf{7}$, p. 57] or Seifert circuits in $[\mathbf{1 2}, \mathrm{p}$. $390]$.

PROPOSITION 4.1. Let $L$ be a nonsplit positive (negative) link in $S^{3}$, and $\tilde{L}$ a positive (negative) diagram of $L$. Let $m(\tilde{L})$ be the number of Seifert circles in $\tilde{L}$ and $\alpha(\tilde{L})$ the number of domains in $S^{2}$ divided by $\tilde{L}$. Then

$$
\begin{aligned}
& \text { (1) } g(L)=\frac{1}{2}\{\delta(L)-\mu(L)+1\} \\
& (2) \quad \delta(L)=\alpha(\tilde{L})-m(\tilde{L})-1 .
\end{aligned}
$$

This proposition was essentially proved in [11], but for the details, see [2].

Now to each double point $c$, four (not necessarily distinct) domains are incident. If all domains are distinct, $c$ will be called proper. Otherwise, $c$ is called removable. Note that if $c$ is removable, then we can "remove" $c$ from $\tilde{L}$ without changing its link type.

PROPOSITION 4.2. Let $\tilde{L}$ be a positive link diagram with $\lambda(=\lambda(\tilde{L}))$ connected components $\tilde{L}_{1}, \tilde{L}_{2}, \ldots, \tilde{L}_{\lambda}$. Let $\tilde{L}^{*}$ be the diagram obtained from $\tilde{L}$ by eliminating a double point $c$ on $\tilde{L}$. Then

(1) $\tilde{L}^{*}$ is a positive diagram. Let $\tilde{L}_{1}^{*}, \tilde{L}_{2}^{*}, \ldots, \tilde{L}_{\lambda^{*}}^{*}$ be connected components of $\tilde{L}^{*}$.

(2) (i) If $c$ is proper, then $\lambda^{*}=\lambda$, and

$$
\sum_{i=1}^{\lambda} \delta\left(\tilde{L}_{i}\right)=\sum_{i=1}^{\lambda} \delta\left(\tilde{L}_{i}^{*}\right)+1 .
$$

(ii) If $c$ is removable, then $\lambda^{*}=\lambda+1$, and

$$
\sum_{i=1}^{\lambda} \delta\left(\tilde{L}_{i}\right)=\sum_{i=1}^{\lambda+1} \delta\left(L_{i}^{*}\right) .
$$




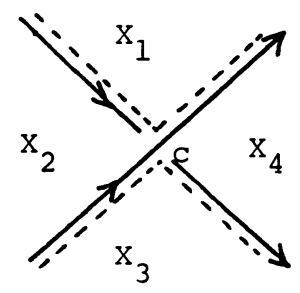

FIGURE 3

Proof. (1) is obvious. To prove (2), let $X_{1}, X_{2}, X_{3}, X_{4}$ be four domains meeting at $c$ (see Figure 3).

Suppose $c$ is proper. Then $X_{2}$ and $X_{4}$ are distinct domains and $\lambda\left(\tilde{L}^{*}\right)=\lambda(\tilde{L})$. But the elimination of $c$ makes $X_{2}$ and $X_{4}$ amalgamate, and hence $\alpha\left(\tilde{L}^{*}\right)=\alpha(\tilde{L})-$ 1 , from which (4.2) follows by using (4.1)(2). If $c$ is a removable double point on $\tilde{L}_{\lambda}$, say, then $\lambda\left(L^{*}\right)=\lambda(\tilde{L})+1$, and $\tilde{L}_{\lambda}$ splits into two diagrams $\tilde{L}_{\lambda}^{*}$ and $\tilde{L}_{\lambda+1}^{*}$. Obviously $\tilde{L}_{\lambda}$ is a (Schubert) product of two links $L_{\lambda}^{*}$ and $L_{\lambda+1}^{*}$, and hence $\delta\left(L_{\lambda}\right)=\delta\left(L_{\lambda}^{*}\right)+\delta\left(L_{\lambda+1}^{*}\right)$. Since $\delta\left(L_{i}\right)=\delta\left(L_{i}^{*}\right)$ for $i=1,2, \ldots, \lambda-1,(4.3)$ follows immediately.

We generalize Proposition 4.2 to the following.

Proposition 4.3. Let $\tilde{L}$ be a connected positive link diagram. Suppose we obtain $\lambda$ connected diagrams $\tilde{L}_{1}, \tilde{L}_{2}, \ldots, \tilde{L}_{\lambda}$ by eliminating d double points on $\tilde{L}$. Then

$$
\sum_{i=1}^{\lambda} \delta\left(\tilde{L}_{i}\right)-\lambda+d+1=\delta(\tilde{L}) .
$$

ProOF. A proof will be by induction on $d$.

If $d=1$, then $\lambda=1$ or 2 and Proposition 4.3 follows from Proposition 4.2.

Inductively, suppose (4.4) is true for $j<d$. Let $\tilde{L}^{\prime}$ be the link diagram obtained from $\tilde{L}$ by eliminating $d-1$ double points $c_{1}, c_{2}, \ldots, c_{d-1}$ on $\tilde{L}$. Suppose $\tilde{L}^{\prime}$ has $\lambda^{\prime}$ connected components $\tilde{L}_{1}^{\prime}, \ldots, \tilde{L}_{\lambda^{\prime}}^{\prime}$. We may assume inductively

$$
\sum_{i=1}^{\lambda^{\prime}} \delta\left(\tilde{L}_{i}^{\prime}\right)-\lambda^{\prime}+(d-1)+1=\delta(\tilde{L}) .
$$

Now we eliminate $c_{d}$ so that we have a link diagram $\tilde{L}^{\prime \prime}$ of $\lambda^{\prime \prime}$ connected components $\tilde{L}_{1}^{\prime \prime}, \ldots, \tilde{L}_{\lambda^{\prime \prime}}^{\prime \prime}$.

If $\lambda^{\prime \prime}=\lambda^{\prime}$, then $\sum_{i=1}^{\lambda^{\prime \prime}} \delta\left(L_{i}^{\prime \prime}\right)+1=\sum_{i=1}^{\lambda^{\prime}} \delta\left(L_{i}^{\prime}\right)$, and hence, by (4.5), we have

$$
\begin{aligned}
\delta(\tilde{L}) & =\sum_{i=1}^{\lambda^{\prime}} \delta\left(L_{i}^{\prime}\right)-\lambda^{\prime}+d=\sum_{i=1}^{\lambda^{\prime \prime}} \delta\left(L_{i}^{\prime \prime}\right)+1-\lambda^{\prime}+d \\
& =\sum_{i=1}^{\lambda^{\prime \prime}} \delta\left(L_{i}^{\prime \prime}\right)-\lambda^{\prime \prime}+d+1
\end{aligned}
$$


If $\lambda^{\prime \prime}=\lambda^{\prime}+1$, then (4.3) and (4.5) imply

$$
\begin{aligned}
\delta(\tilde{L}) & =\sum_{i=1}^{\lambda^{\prime}} \delta\left(L_{i}^{\prime}\right)-\lambda^{\prime}+d=\sum_{i=1}^{\lambda^{\prime}+1} \delta\left(L_{i}^{\prime \prime}\right)-\left(\lambda^{\prime}+1\right)+(d+1) \\
& =\sum_{i=1}^{\lambda^{\prime \prime}} \delta\left(L_{i}^{\prime \prime}\right)-\lambda^{\prime \prime}+d+1 .
\end{aligned}
$$

The induction is now completed.

5. Proof of Theorem 2.1 (III). Let $\tilde{L}$ be a connected positive link diagram of a positive link $L$. Let $c_{1}, c_{2}, \ldots, c_{k}$ be double points on $\tilde{L}$ to which we apply the fundamental identity (2.1).

In this section, we will prove a reduction formula (5.5) to compute $J_{L}(t)$.

Now first we change the incident sign at $c_{1}$ to get $\tilde{L}_{1}$. Since $\varepsilon\left(c_{1}\right)=+1$, the fundamental identity (2.1) gives us

$$
(-1) P(L ; l, m)=l^{2} P\left(L_{1} ; l, m\right)+l m P\left(L_{1}^{*} ; l, m\right),
$$

where $L_{1}^{*}$ is the link diagram obtained from $\tilde{L}$ by eliminating $c_{1}$ from $\tilde{L}$. Note that $\tilde{L}_{1}^{*}$ is again a positive diagram. Next, change the incident sign at $c_{2}$ on $\tilde{L}_{1}$. Then the fundamental identity (2.1) tells us

$$
(-1) P\left(L_{1} ; l, m\right)=l^{2} P\left(L_{1,2} ; l, m\right)+l m P\left(\hat{L}_{1,2} ; l, m\right),
$$

where $L_{1,2}$ is the link obtained from $L_{1}$ by changing the incident sign at $c_{2}$ and $\hat{L}_{1,2}$ is the link obtained from $L_{1}$ by eliminating $c_{2}$. Since $L_{1}$ is not a positive diagram, $\hat{L}_{1,2}$ is not a positive diagram.

To evaluate $P\left(\hat{L}_{1,2} ; l, m\right)$, we change the incident sign at $c_{1}$ on $\hat{L}_{1,2}$ again, and apply the fundamental formula (2.1) to obtain

$$
(-1) P\left(\hat{L}_{1,2} ; l, m\right)=l^{-2} P\left(L_{2}^{*} ; l, m\right)+l^{-1} m P\left(L_{1,2}^{*} ; l, m\right),
$$

where $\tilde{L}_{2}^{*}$ is the diagram obtained from $\tilde{L}$ by eliminating $c_{2}$, and $\tilde{L}_{1,2}^{*}$ is the diagram obtained from $\tilde{L}$ by elimimating $c_{1}$ and $c_{2}$. Both $\tilde{L}_{2}^{*}$ and $\tilde{L}_{1,2}^{*}$ are positive diagrams. Substituting (5.3) into (5.2), we obtain (5.4) from (5.1):

$$
\begin{aligned}
(-1)^{2} P(L ; l, m)= & (-1) l^{2} P\left(L_{1} ; l, m\right)+(-1) l m P\left(L_{1}^{*} ; l, m\right) \\
= & l^{4} P\left(L_{1,2} ; l, m\right)+l^{3} m P\left(\hat{L}_{1,2} ; l, m\right)+(-1) l m P\left(L_{1}^{*} ; l, m\right) \\
= & l^{4} P\left(L_{1,2} ; l, m\right)+(-1) l^{3} m\left\{l^{-2} P\left(L_{2}^{*} ; l, m\right)+l^{-1} m P\left(L_{1,2}^{*} ; l, m\right)\right\} \\
& +(-1) l m P\left(L_{1}^{*} ; l, m\right) \\
= & l^{4} P\left(L_{1,2} ; l, m\right)+(-1) l m\left\{P\left(L_{1}^{*} ; l, m\right)+P\left(L_{2}^{*} ; l, m\right)\right\} \\
& +(-1) l^{2} m^{2} P\left(L_{1,2}^{*} ; l, m\right) .
\end{aligned}
$$

Now an easy induction argument proves the basic reduction formula

$$
\begin{aligned}
P(L ; l, m)= & (-1)^{k} l^{2 k} P\left(L_{1,2, \ldots, k} ; l, m\right) \\
& +(-1) \sum_{d=1}^{k}(l m)^{d} \sum_{1 \leq j_{1}<\cdots<j_{d} \leq k} P\left(L_{j_{1}, \ldots, j_{d}}^{*} ; l, m\right),
\end{aligned}
$$


where $\tilde{L}_{j_{1}, \ldots, j_{d}}^{*}$ is a positive diagram obtained from $\tilde{L}$ by eliminating $d$ double points $c_{j_{1}}, \ldots, c_{j_{d}}$, and $\tilde{L}_{1,2, \ldots, k}$ is a diagram obtained from $\tilde{L}$ by changing the incident signs at $c_{1}, c_{2}, \ldots, c_{k}$.

Since $L$ has $n$-components, it follows from (5.5) that

$$
\begin{aligned}
Q(L ; l, m)= & \left(\frac{m}{l}\right)^{n-1} P(L ; l, m) \\
= & (-1)^{k}\left(\frac{m}{l}\right)^{n-1} l^{2 k} P\left(L_{1,2, \ldots, k} ; l, m\right) \\
& +(-1) \sum_{d=1}^{k}(l m)^{d}\left(\frac{m}{l}\right)^{n-1} \sum_{1 \leq j_{1}<\cdots<j_{d} \leq k} P\left(L_{j_{1}, \ldots, j_{d}}^{*} ; l, m\right) \\
= & (-1)^{k} l^{2 k} Q\left(L_{1,2, \ldots, k} ; l, m\right) \\
& +(-1) \sum_{d=1}^{k}(l m)^{d}\left(\frac{m}{l}\right)^{n-1} \sum_{1 \leq j_{1}<\cdots<j_{d} \leq k} P\left(L_{j_{1}, \ldots, j_{d}}^{*} ; l, m\right) .
\end{aligned}
$$

In the next section, we will evaluate $l$-deg, $l$-coef and $r$-deg of each term in the second summation in (5.6).

6. Proof of Theorem 2.1 (IV). Let

$$
R\left(j_{1}, \ldots, j_{d}\right)=(l m)^{d}(m / l)^{n-1} P\left(L_{j_{1}, \ldots, j_{d}}^{*} ; l, m\right) .
$$

By the substitutions $l=t \sqrt{-1}$ and $m=-(\sqrt{t}-1 / \sqrt{t}) \sqrt{-1}, R\left(j_{1}, \ldots, j_{d}\right)$ becomes an integer polynomial $R^{*}\left(j_{1}, \ldots, j_{d}\right)$ in $t^{ \pm 1}$. In this section we will show that $l-\operatorname{deg} R^{*}\left(j_{1}, \ldots, j_{d}\right)$ depends on $d$, not a sequence $j_{1}, j_{2}, \ldots, j_{d}$. In fact, we will prove the following lemma.

LEMMA 6.1. For any $1 \leq d \leq k$ and $1 \leq j_{1}<\cdots<j_{d} \leq k$,

(1) $l$-coef $R^{*}\left(j_{1}, \ldots, j_{d}\right)=(-1)^{d}$;

(2) $l-\operatorname{deg} R^{*}\left(j_{1}, \ldots, j_{d}\right)=\frac{1}{2}\{\delta(L)-n+1\}-(n-1)$

$$
=g(L)-(n-1)
$$

(3) if $L_{j_{1}, \ldots, j_{d}}^{*}$ is not split, then

$$
r-\operatorname{deg} R^{*}\left(j_{1}, \ldots, j_{d}\right) \leq h(\tilde{L})+(n-1) .
$$

Note that $n=\mu(L)$.

ProOF. Suppose $\tilde{L}^{*}=\tilde{L}_{j_{1}, \ldots, j_{d}}^{*}$ consists of $\lambda$ connected components $\tilde{L}_{1}, \ldots, \tilde{L}_{\lambda}$. Since the number of components of a link increases or decreases by one, each time we eliminate a double point of a link diagram, we see that $\mu\left(L^{*}\right) \equiv n-d(\bmod 2)$, and hence we can write $\mu\left(L^{*}\right)=n-d+2 q$ for some integer $q$. Therefore,

$$
\sum_{i=1}^{\lambda}\left\{\mu\left(L_{i}\right)-1\right\}=\mu\left(L^{*}\right)-\lambda=n-d+2 q-\lambda .
$$

Since

$$
P\left(L^{*} ; l, m\right)=\left(-\frac{l+l^{-1}}{m}\right)^{\lambda-1} \prod_{p=1}^{\lambda} P\left(L_{p} ; l, m\right)
$$


and

$$
Q\left(L_{p} ; l, m\right)=(m / l)^{n_{p}-1} P\left(L_{p} ; l, m\right), \quad n_{p}=\mu\left(L_{p}\right),
$$

for $p=1,2, \ldots, \lambda$, we have

$$
\begin{aligned}
R\left(j_{1}, \ldots, j_{d}\right)= & (l m)^{d}\left(\frac{m}{l}\right)^{n-1}\left(-\frac{l+l^{-1}}{m}\right)^{\lambda-1} \prod_{p=1}^{\lambda} P\left(L_{p} ; l, m\right) \\
= & (l m)^{d}\left(\frac{m}{l}\right)^{n-1}\left(-\frac{l+l^{-1}}{m}\right)^{\lambda-1} \\
& \times \prod_{p=1}^{\lambda}\left\{\left(\frac{m}{l}\right)^{n_{p}-1} P\left(L_{p} ; l, m\right)\right\} \prod_{p=1}^{\lambda}\left(\frac{l}{m}\right)^{n_{p}-1} .
\end{aligned}
$$

Noting $\sum_{p=1}^{\lambda}\left(n_{p}-1\right)=n-d+2 q-\lambda$, we see

$$
\begin{aligned}
R\left(j_{1}, \ldots, j_{d}\right) & =(l m)^{d}\left(\frac{m}{l}\right)^{n-1}\left(\frac{l}{m}\right)^{n-d+2 q-\lambda}\left(-\frac{l+l^{-1}}{m}\right)^{\lambda-1} \prod_{p=1}^{\lambda} Q\left(L_{p} ; l, m\right) \\
& =(l m)^{d}\left(\frac{m}{l}\right)^{d-2 q+\lambda-1}(-1)^{\lambda-1}\left(\frac{l+l^{-1}}{m}\right)^{\lambda-1} \prod_{p=1}^{\lambda} Q\left(L_{p} ; l, m\right) \\
& =(-1)^{\lambda-1}(l m)^{d}\left(\frac{m}{l}\right)^{d-2 q}\left(1+l^{-2}\right)^{\lambda-1} \prod_{p=1}^{\lambda} Q\left(L_{p} ; l, m\right) \\
& =(-1)^{\lambda-1} m^{2(d-q)} l^{2 q}\left(1+l^{-2}\right)^{\lambda-1} \prod_{p=1}^{\lambda} Q\left(L_{p} ; l, m\right) .
\end{aligned}
$$

Therefore, we obtain

(6.3)

$$
\begin{aligned}
R^{*}\left(j_{1}, \ldots, j_{d}\right) & =(-1)^{\lambda-1}(-1)^{d-q}(-1)^{q}\left(t-2+t^{-1}\right)^{d-q} t^{2 q}\left(1-t^{-2}\right)^{\lambda-1} \prod_{p=1}^{\lambda} J\left(L_{p} ; t\right) \\
& =(-1)^{\lambda+d-1}\left(t-2+t^{-1}\right)^{d-q} t^{2 q}\left(1-t^{-2}\right)^{\lambda-1} \prod_{p=1}^{\lambda} J\left(L_{p} ; t\right) .
\end{aligned}
$$

Since $h\left(\tilde{L}_{p}\right) \leq h(\tilde{L})-d<h(\tilde{L})$ and $\tilde{L}_{p}$ is connected, we can apply the induction hypothesis on each reduced Jones polynomial $J\left(L_{p} ; t\right)$, and we have

For each $p=1,2, \ldots, \lambda$,

(1) $l$-coef $J\left(L_{p} ; t\right)=1$;

(2) $l-\operatorname{deg} J\left(L_{p} ; t\right)=\frac{1}{2}\left\{\delta\left(L_{p}\right)-n_{p}+1\right\}-\left(n_{p}-1\right)$;

(3) $r-\operatorname{deg} J\left(L_{p} ; t\right) \leq h\left(\tilde{L}_{p}\right)+\left(n_{p}-1\right)$.

Therefore

$$
l \text {-coef } R^{*}\left(j_{1}, \ldots, j_{d}\right)=(-1)^{\lambda+d-1}(-1)^{\lambda-1} \prod_{p=1}^{\lambda} l-\operatorname{coef} J\left(L_{p} ; t\right)=(-1)^{d} .
$$

This proves $(6.1)(1)$. 
To prove (6.1)(2), first we see from (6.3) that

$$
\begin{aligned}
l-\operatorname{deg} R^{*}\left(j_{1}, \ldots, j_{d}\right) & =-(d-q)+2 q-2(\lambda-1)+\sum_{p=1}^{\lambda} l-\operatorname{deg} J\left(L_{p} ; t\right) \\
& =-d+3 q-2(\lambda-1)+\sum_{p=1}^{\lambda}\left[\frac{1}{2}\left\{\delta\left(L_{p}\right)-n_{p}+1\right\}-\left(n_{p}-1\right)\right] \\
& =\frac{1}{2}\left\{\sum_{p=1}^{\lambda} \delta\left(L_{p}\right)-3 \sum_{p=1}^{\lambda} n_{p}+3 \lambda-2 d+6 q-4 \lambda+4\right\} \\
& =\frac{1}{2}\left\{\sum_{p=1}^{\lambda} \delta\left(L_{p}\right)-3(n-d+2 q)-2 d+6 q-\lambda+4\right\} \\
& =\frac{1}{2}\left\{\sum_{p=1}^{\lambda} \delta\left(L_{p}\right)-3 n+d-\lambda+4\right\} .
\end{aligned}
$$

Now Proposition 4.3 (4.4) shows that $\sum_{p=1}^{\lambda} \delta\left(L_{p}\right)-\lambda+d+1=\delta(L)$ and hence

$$
l-\operatorname{deg} R^{*}\left(j_{1}, \ldots, j_{d}\right)=\frac{1}{2}\{\delta(L)-3 n+3\}=\frac{1}{2}\{\delta(L)-n+1\}-(n-1) .
$$

This proves $(6.1)(2)$.

Finally, suppose $L^{*}=L_{j_{1}, \ldots, j_{d}}^{*}$ is connected, and we will prove (6.1)(3). Putting $\lambda=1$ in (6.3), we have

$$
h-\operatorname{deg} R^{*}\left(j_{1}, \ldots, j_{d}\right)=d-q+2 q+h-\operatorname{deg} J\left(L^{*} ; t\right)=d+q+h-\operatorname{deg} J\left(L^{*} ; t\right) .
$$

Since $l-\operatorname{deg} R^{*}\left(j_{1}, \ldots, j_{d}\right)=l-\operatorname{deg} J\left(L^{*} ; t\right)+3 q-d$, it follows that

$$
r-\operatorname{deg} R^{*}\left(j_{1}, \ldots, j_{d}\right)=r-\operatorname{deg} J\left(L^{*} ; t\right)+2 d-2 q \text {. }
$$

Since $h\left(\tilde{L}^{*}\right)=h(\tilde{L})-d<h(\tilde{L})$, we can apply the induction hypothesis to obtain

$$
r-\operatorname{deg} J\left(L^{*} ; t\right) \leq h\left(\tilde{L}^{*}\right)+\left(\mu\left(L^{*}\right)-1\right)
$$

and hence

$$
\begin{aligned}
r-\operatorname{deg} R^{*}\left(j_{1}, \ldots, j_{d}\right) & \leq h\left(\tilde{L}^{*}\right)+\mu\left(L^{*}\right)-1+2 d-2 q \\
& =h\left(\tilde{L}^{*}\right)+n-d+2 q-1+2 d-2 q \\
& =h\left(\tilde{L}^{*}\right)+d+(n-1)=h(\tilde{L})+(n-1) .
\end{aligned}
$$

This proves $(6.1)(3)$.

7. Proof of Theorem 2.1 (V). In order to apply the induction hypothesis on the reduction formula (5.5), we are required to show that

$$
L_{1,2, \ldots, k} \text { is isotropic to a positive link } L^{\prime} \text { such that } h\left(\tilde{L}^{\prime}\right)<h(\tilde{L}) \text {. }
$$

For an arbitrary choice of double points $c_{1}, c_{2}, \ldots, c_{k},(7.1)$, in general, is not true. In this section, however, we will show that there exist double points $c_{1}, \ldots, c_{k}$ 


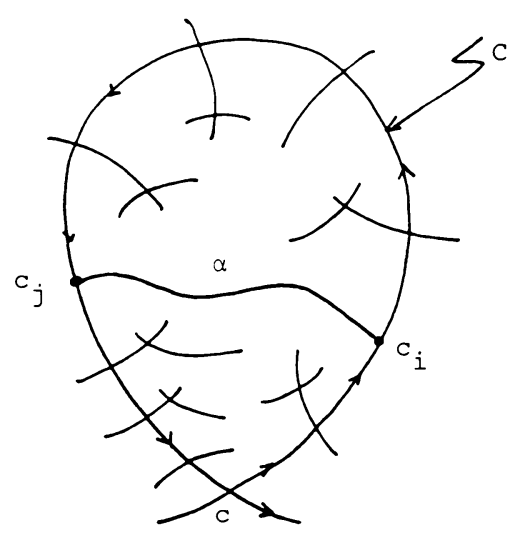

FIGURE 4

for which (7.1) holds. In fact we will prove

LEMMA 7.1. Let $L$ be a nonsplit positive link and $\tilde{L}$ a connected positive link diagram of $L$. Then there are double points $c_{1}, c_{2}, \ldots, c_{k}$ on $\tilde{L}$ such that

(1) $L_{1,2, \ldots, k}$ is isotopic to a link $L^{\prime}$ such that $h\left(\tilde{L}^{\prime}\right)=h(\tilde{L})-2 k$, and

(2) for any sequence $j_{1}, \ldots, j_{d}, 1 \leq d \leq k, 1 \leq j_{1}<j_{2}<\cdots<j_{d} \leq k, L_{j_{1}, \ldots, j_{d}}^{*}$ is connected.

PROOF. A proof is based upon a standard geometric argument.

Take a component $L_{1}$, say, of a link $L$. It is then easy to see that $L_{1}$ has two points $P$ and $P^{\prime}\left(P\right.$ may be $\left.P^{\prime}\right)$ such that the part of $L_{1}$ joining $P$ and $P^{\prime}$ projects on a circle $C$ in $\tilde{L}$ and such that $P$ and $P^{\prime}$ project on a double point $c$ of $\tilde{L}$. Since $\tilde{L}$ is connected, such $P$ and $P^{\prime}$ exist. If $P=P^{\prime}$, then $L_{1}$ is unknotted.

Now the circle $C$ divides $S^{2}$ into two domains. The left side domain of $C$ (with respect to its direction) is called the interior of $C$, denoted by $\bar{C}$.

First we consider the case $P \neq P^{\prime}$.

Let $c$ be the double point of $\tilde{L}$ on which $P$ and $P^{\prime}$ project. If $C$ has no other double points, then $c$ is removable, and hence we can remove $c$ from $\tilde{L}$ without changing the link type $L$, and there is nothing to prove here.

Suppose $C$ contains $m$ double points $c_{1}, c_{2}, \ldots, c_{m}$ besides $c . m$ is obviously even. A simple arc $\alpha$ joining two double points $c_{i}$ and $c_{j}(1 \leq i<j \leq m)$ in $\bar{C}$ is called a separating arc of $C$ if (1) $\alpha$ does not intersect with $\tilde{L}$ except its end points, and (2) each of two domains into which $\alpha$ divides $\bar{C}$ has the nonempty intersection with $\tilde{L}$ except its end points (see Figure 4 ).

Let $\alpha$ be a separating arc of $C$ and $\alpha$ joins $c_{i}$ and $c_{j}, 1 \leq i<j \leq m$. $\alpha$ divides $C$ into two arcs $\alpha_{+}$and $\alpha_{-}$, where $\alpha_{+}$contains $c_{i}, c_{i+1}, \ldots, c_{j}$ and $\alpha_{-}$contains $c_{j}$, $c_{j+1}, \ldots, c_{m}, c, c_{1}, \ldots, c_{i-1}, c_{i}$.

Now $\alpha$ is called the innermost separating arc of $C$ if the subdomain of $\bar{C}$ bounded by $\alpha_{+}$and $\alpha$ does not have any other separating $\operatorname{arcs}$ of $C$.

If $C$ has separating arcs, $C$ must have at least one innermost separating arc. Let $\beta$ be an innermost separating arc and suppose $\beta$ joins $c_{i}$ and $c_{j}, 1 \leq i<j \leq m$. Then $j-i$ must be even; i.e., $\beta_{+}$contains an even number of double points. Since $\tilde{L}$ is positive, half of these double points are overcrossing points on $C$ including 


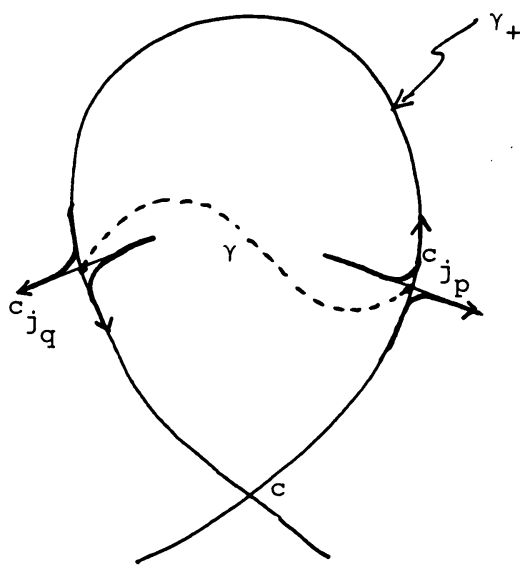

FIGURE 5

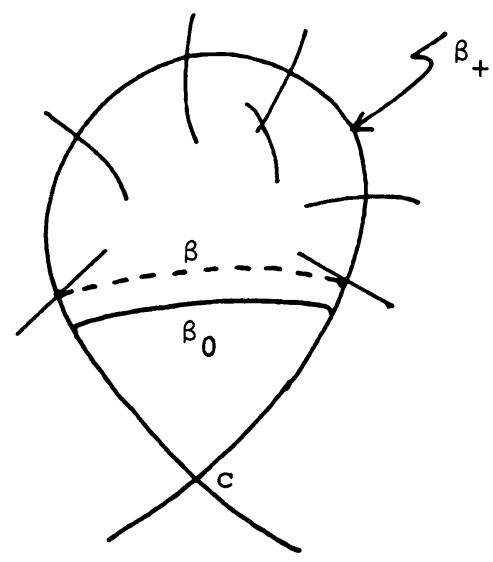

FIGURE 6

possibly $c_{i}$ and $c_{j}$. These double points are exactly what we are looking for. If $C$ has no separating arcs, then all the overcrossing double points on $C$ are what we sought. In fact, if $L_{j_{1}, \ldots, j_{d}}^{*}$ is disconnected, then there is a separating arc $\gamma$ in $C$ which joins overcrossing double points $c_{j_{p}}$ and $c_{j_{q}}$, say. Since $\beta$ is innermost, $\gamma$ must be $\beta$. However, $\gamma_{+}$contains an odd number of double points as is seen in Figure 5 , while $\beta_{+}$contains an even number of double points, a contradiction.

When $P=P^{\prime}$, then we include $c$ in the sequence of double points $c_{1}, c_{2}, \ldots, c_{m}$, and apply a similar argument.

Obviously, $L_{1,2, \ldots, k}$ is isotopic to $L^{\prime}$ with $h\left(\tilde{L}^{\prime}\right)=h(\tilde{L})-2 k$ by pulling $\beta_{+}$down to $\beta_{0}$ (Figure 6).

This proves Lemma 7.1.

8. Proof of Theorem 2.1 (VI). We return to the proof of Theorem 2.1.

We may assume now that $L_{j_{1}, \ldots, j_{d}}^{*}$ is connected and $L_{1,2, \ldots, k}$ is isotopic to $L^{\prime}$ such that $h\left(\tilde{L}^{\prime}\right) \leq h(\tilde{L})-2 k, k \geq 1$. 
Consider $Q_{L}(l, m)$ or $J_{L}(t)$. We proved in Lemma 6.1 that $l$-deg of each term in the second summation of (5.6) is always equal to $g(L)-(n-1)$ and its $l$-coef is $(-1)^{d}$. Since there are exactly $\left(\begin{array}{l}k \\ d\end{array}\right)$ choices of sequences $j_{1}, \ldots, j_{d}$ from $k$ distinct indices $1,2, \ldots, k$, the coefficient of the term $t^{g(L)-n+1}$ in the second term of (5.6) is

$$
(-1) \sum_{d=1}^{k}\left(\begin{array}{l}
k \\
d
\end{array}\right)(-1)^{d}=+1 .
$$

Now the proof of Theorem 2.1 will be completed by evaluating $l$-deg and $h$-deg of the first and second terms of (5.6).

In order to prove Theorem 2.1, therefore, it is enough to show

$$
\begin{aligned}
& \text { (1) } 2 k+l-\operatorname{deg} J_{L^{\prime}}(t)>g(L)-(n-1) ; \\
& \text { (2) } 2 k+h-\operatorname{deg} J_{L^{\prime}}(t)-\{g(L)-(n-1)\} \leq h(\tilde{L})+(n-1) \text {. }
\end{aligned}
$$

Note that $\tilde{L}$ is connected.

Suppose $\tilde{L}^{\prime}$ consists of $\lambda$ connected positive link diagrams $\tilde{L}_{1}^{\prime}, \tilde{L}_{2}^{\prime}, \ldots, \tilde{L}_{\lambda}^{\prime}$. However, the construction of $L_{1,2, \ldots, k}$ given in $\S 7$ shows implicitly that $\lambda=1$ or 2 . Therefore, we assume, henceforth, $\lambda \leq 2$. Let $J_{i}(t)$ denote the reduced Jones polynomial of $L_{i}^{\prime}, 1 \leq i \leq \lambda$. Since $\sum_{i=1}^{\lambda} h\left(\tilde{L}_{i}^{\prime}\right)+2 k=h(\tilde{L})$ and $k \geq 1$, it follows that $h\left(\tilde{L}_{i}^{\prime}\right)<h(\tilde{L})$; therefore by the induction hypothesis

$$
\begin{aligned}
& \text { (1) } \quad l-\operatorname{deg} J_{i}(t)=g\left(L_{i}^{\prime}\right)-\left\{\mu\left(L_{i}^{\prime}\right)-1\right\} \\
& \text { (2) } \quad r-\operatorname{deg} J_{i}(t) \leq h\left(\tilde{L}_{i}^{\prime}\right)+\left\{\mu\left(L_{i}^{\prime}\right)-1\right\} .
\end{aligned}
$$

Since $\tilde{L}_{1}^{\prime}, \ldots, \tilde{L}_{\lambda}^{\prime}$ are connected components of $\tilde{L}^{\prime}$, it follows from Proposition 3.1 that

$$
\begin{aligned}
l-\operatorname{deg} J_{L^{\prime}}(t) & =\sum_{i=1}^{\lambda} l-\operatorname{deg} J_{i}(t)-2(\lambda-1) \\
& =\sum_{i=1}^{\lambda}\left\{g\left(L_{i}^{\prime}\right)-\mu\left(L_{i}^{\prime}\right)+1\right\}-2(\lambda-1) \\
& =\sum_{i=1}^{\lambda} g\left(L_{i}^{\prime}\right)-n+\lambda-2(\lambda-1) \\
& =\sum_{i=1}^{\lambda} g\left(L_{i}^{\prime}\right)-(n-1)-(\lambda-1), \\
\text { (2) } r-\operatorname{deg} J_{L^{\prime}}(t) & \leq h\left(\tilde{L}^{\prime}\right)+(n-1)+(\lambda-1) .
\end{aligned}
$$

Therefore, $(8.1)(1)$ is equivalent to

$$
\sum_{i=1}^{\lambda} g\left(L_{i}^{\prime}\right)-(\lambda-1)+2 k>g(L) .
$$

Now let $\alpha(\tilde{X})$ and $s(\tilde{X})$ denote, respectively, the number of domains and Seifert circles in a link diagram $\tilde{X}$. Then Proposition 4.1 and a simple computation show 
that (8.4) is equivalent to

$$
4 k+s(\tilde{L})-\sum_{i=1}^{\lambda} s\left(\tilde{L}_{i}^{\prime}\right)>\alpha(\tilde{L})-\sum_{i=1}^{\lambda} \alpha\left(\tilde{L}_{i}^{\prime}\right)+2(\lambda-1) .
$$

Furthermore, (8.3)(2) implies that

$$
\begin{aligned}
2 k & +h-\operatorname{deg} J_{L^{\prime}}(t)-\{g(L)-n+1\} \\
& =2 k+r-\operatorname{deg} J_{L^{\prime}}(t)+l-\operatorname{deg} J_{L^{\prime}}(t)-\{g(L)-n+1\} \\
& \leq 2 k+h\left(\tilde{L}^{\prime}\right)+(n-1)+(\lambda-1)+l-\operatorname{deg} J_{L^{\prime}}(t)-g(L)+(n-1) \\
& =h(\tilde{L})+2(n-1)+(\lambda-1)+l-\operatorname{deg} J_{L^{\prime}}(t)-g(L) .
\end{aligned}
$$

Therefore, in order to prove $(8.1)(2)$, it suffices to show that

$$
h(\tilde{L})+2(n-1)+(\lambda-1)+l-\operatorname{deg} J_{L^{\prime}}(t)-g(L) \leq h(\tilde{L})+(n-1),
$$

or equivalently,

$$
(\lambda-1)+l-\operatorname{deg} J_{L^{\prime}}(t) \leq g(L)-n+1
$$

Using Proposition 4.1 and (8.3)(1), we see that (8.6) is equivalent to

$$
(\lambda-1)+\sum_{i=1}^{\lambda} g\left(L_{i}^{\prime}\right)-(n-1)-(\lambda-1) \leq g(L)-(n-1) .
$$

That is,

$$
\sum_{i=1}^{\lambda} g\left(L_{i}^{\prime}\right) \leq g(L)
$$

Now a simple calculation shows that (8.7) is equivalent to

$$
s(\tilde{L})-\sum_{i=1}^{\lambda} s\left(\tilde{L}_{i}^{\prime}\right) \leq \alpha(\tilde{L})-\sum_{i=1}^{\lambda} \alpha\left(\tilde{L}_{i}^{\prime}\right) .
$$

Therefore the final lemma needed to complete our proof is

LEMMA 8.1 .

$$
\begin{aligned}
& \text { (1) } \quad 4 k+s(\tilde{L})-\sum_{i=1}^{\lambda} s\left(\tilde{L}_{i}^{\prime}\right)>\alpha(\tilde{L})-\sum_{i=1}^{\lambda} \alpha\left(\tilde{L}_{i}^{\prime}\right)+2(\lambda-1) \\
& \text { (2) } \alpha(\tilde{L})-\sum_{i=1}^{\lambda} \alpha\left(\tilde{L}_{i}^{\prime}\right) \geq s(\tilde{L})-\sum_{i=1}^{\lambda} s\left(\tilde{L}_{i}^{\prime}\right)
\end{aligned}
$$

ProOF. We will use the same notation as in the previous section.

For simplicity, we let $s\left(\tilde{L}^{\prime}\right)=\sum_{i=1}^{\lambda} s\left(\tilde{L}_{i}^{\prime}\right)$ and $\alpha\left(\tilde{L}^{\prime}\right)=\sum_{i=1}^{\lambda} \alpha\left(\tilde{L}_{i}^{\prime}\right)$. Since we are only interested in $s(\tilde{L})-s\left(\tilde{L}^{\prime}\right)$ and $\alpha(\tilde{L})-\alpha\left(\tilde{L}^{\prime}\right)$ and since these numbers are affected only when a Seifert circle intersects $\beta_{+}$, we consider only these Seifert circles.

First we suppose $P \neq P^{\prime}$, where $P$ and $P^{\prime}$ are points on one component of $L$ considered in $\S 7$. (For the case $P=P^{\prime}$, the proof is analogous and therefore is omitted.) 


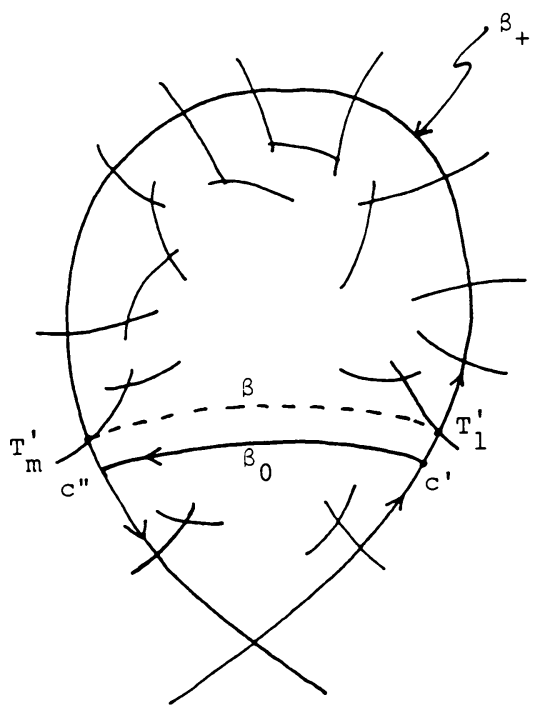

FIGURE 7

Suppose there are $m$ Seifert circles $T_{1}^{\prime}, T_{2}^{\prime}, \ldots, T_{m}^{\prime}$ in $\tilde{L}^{\prime}$ that intersect $\beta_{+}$in $\tilde{L}$. (Beware that $T_{i}^{\prime}$ is a Seifert circle in $\tilde{L}^{\prime}$ but not in $\tilde{L}$.) See Figure 7 .

Let $c^{\prime}$ and $c^{\prime \prime}$ be the end points of $\beta_{0} . c^{\prime}$ and $c^{\prime \prime}$ are not double points of $\tilde{L}$. We extend $\beta_{+}$to the arc $\hat{\beta}_{+}$in $\tilde{L}$ which joins $c^{\prime}$ to $c^{\prime \prime}$. Therefore, $\hat{\beta}_{+}$and $\beta_{0}$ form a simple closed curve in $S^{2}$.

Now when $\beta_{0}$ is replaced by $\hat{\beta}_{+}$, each domain in $\tilde{L}^{\prime}$ is cut into two domains in $\tilde{L}$. Therefore we have

$$
\alpha(\tilde{L})-\alpha\left(\tilde{L}^{\prime}\right)=2 k .
$$

Suppose there are $r$ Seifert circles in $\tilde{L}$, each of which contains at least one edge in $\beta_{+}$. We will show

$$
2 k \geq r-m \geq 0 \text {. }
$$

The first inequality is obvious. To prove the second inequality, note that each Seifert circle $T_{i}^{\prime}$ in $\tilde{L}^{\prime}$ is cut into two distinct Seifert circles $T_{i, 1}$ and $T_{i, 2}$ in $\tilde{L}$. However, one of these Seifert circles, say $T_{i, 1}$, may form one Seifert circle in $\tilde{L}$ with, say, $T_{i+1,1}$ that is obtained from $T_{i+1}^{\prime}$. In any case, we have $r-m \geq 0$. This proves (8.11). See Figure 8, where the broken lines indicate Seifert circles in $\tilde{L}$.

Now, if $\lambda=2$ and $k=1$, then $L$ is a product of three links, $L_{1}, L_{2}$ and the Hopf link $L_{3}$ (see Figure 9). Then Theorem 2.1 follows from the induction assumption. Therefore we may assume that $\lambda=1$, or $\lambda=2$ and $k \geq 2$. Then Lemma 8.1 will follow from (8.10) and (8.11).

Since $\lambda-1<k$ and $\alpha(\tilde{L})-\alpha\left(\tilde{L}^{\prime}\right)=2 k$, it follows that

$$
\begin{aligned}
& 4 k+s(\tilde{L})-s\left(\tilde{L}^{\prime}\right)-\left\{\alpha(\tilde{L})-\alpha\left(\tilde{L}^{\prime}\right)+2(\lambda-1)\right\} \\
& \quad=4 k+r-m-2 k-2(\lambda-1) \geq 2 k-2(\lambda-1)>0 .
\end{aligned}
$$

This proves (8.9)(1). Furthermore,

$$
\alpha(\tilde{L})-\alpha\left(\tilde{L}^{\prime}\right)-\left\{s(\tilde{L})-s\left(\tilde{L}^{\prime}\right)\right\}=2 k-(r-m) \geq 0 .
$$

This proves (8.9)(2).

The proof of Theorem 2.1 is now completed. 


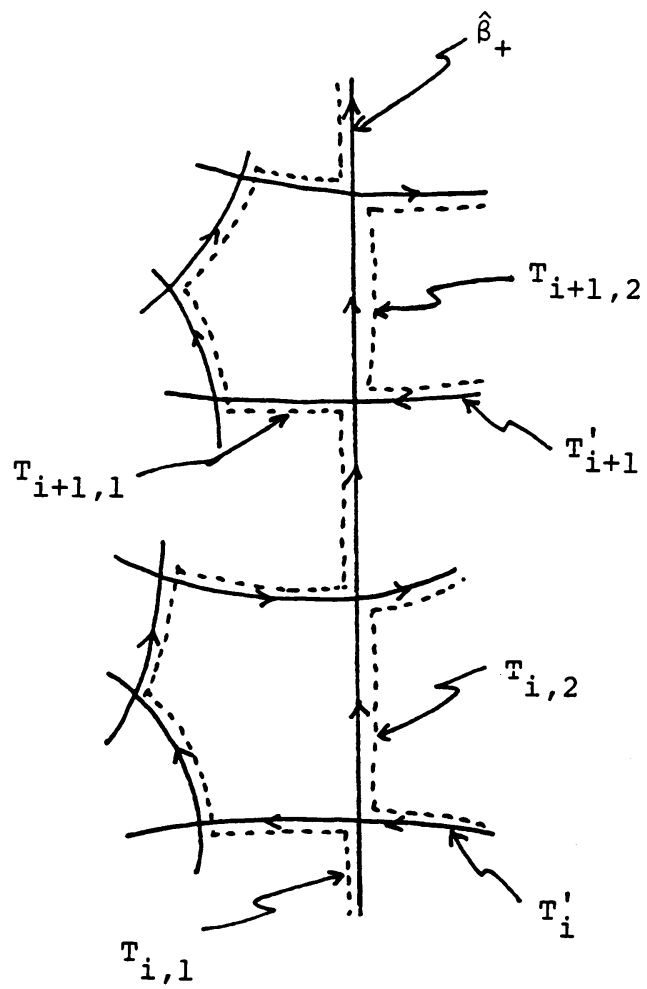

FiguRe 8
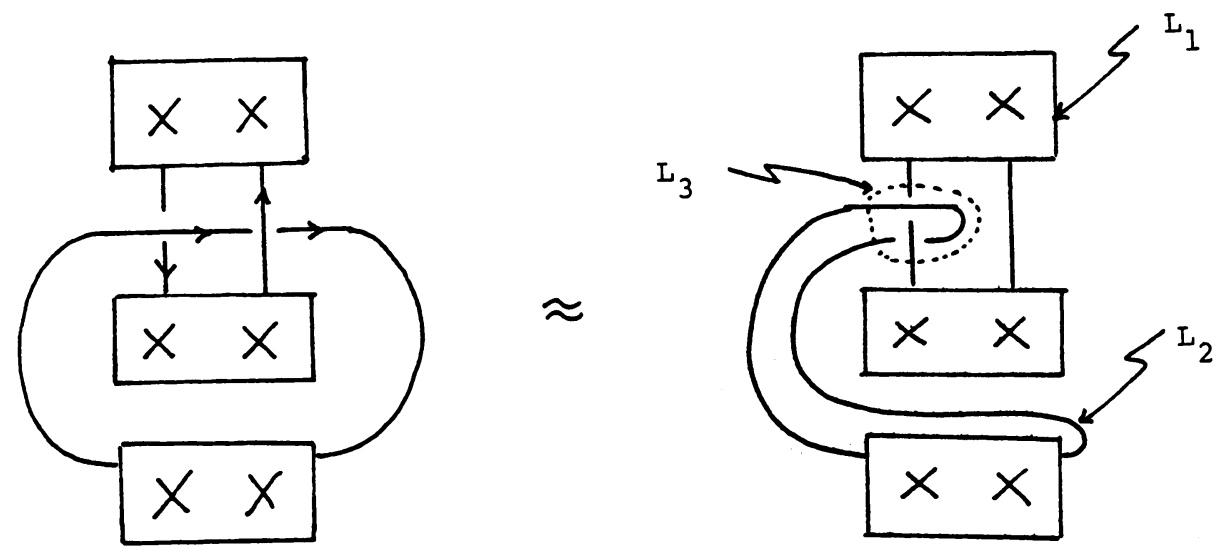

FIGURE 9

9. Alternative links. Let $L_{1}, L_{2}, \ldots, L_{r}$ be $r$ links in $S^{3}$. Denote by $L=$ $L_{1} * L_{2} * \cdots * L_{r}$ the $*$-product (or Murasugi sum) of these links. (For definition, see [4 or 12].) Since $L$ is constructed from $L_{i}$, a diagram $\tilde{L}$ of $L$ is naturally obtained from those of the $L_{i}$ 's, and hence we can write $\tilde{L}=\tilde{L}_{1} * \tilde{L}_{2} * \cdots * \tilde{L}_{r}$. 
Proposition 9.1.

$$
\begin{aligned}
& \text { (1) } h(\tilde{L})=\sum_{i=1}^{r} h\left(\tilde{L}_{i}\right) \\
& \text { (2) } s(L)=\frac{1}{2}\left[\sum_{i=1}^{r}\left\{\mu\left(L_{i}\right)-1\right\}-\{\mu(L)-1\}\right]
\end{aligned}
$$

is an integer.

PROOF. (1) follows from the definition of the *-product. (2) is probably well known. However, here is a quick proof. First span each $L_{i}$ by an orientable surface $F_{i}$. Then the Euler characteristic $\chi\left(F_{i}\right) \equiv \mu\left(L_{i}\right)(\bmod 2)$. The construction of the *-product gives us naturally an orientable spanning surface $F$ of $L$ so that $\chi(F) \equiv \sum_{i=1}^{r} \chi\left(F_{i}\right)-(r-1)(\bmod 2)$. Therefore $\mu(L) \equiv \sum_{i=1}^{r} \mu\left(L_{i}\right)-(r-1)$ $(\bmod 2)$ Q.E.D.

Now let $L_{i}, i=1,2, \ldots, p$, and $L_{j}^{\prime}, j=1,2, \ldots, n$, be, respectively, positive and negative links in $S^{3}$. The $*$-product $L=L_{1} * \cdots * L_{p} * L_{1}^{\prime} * \cdots * L_{n}^{\prime}$ is called an alternative link [7]. In the following, we always assume that $L$ is not split. Let $\tilde{L}=\tilde{L}_{1} * \cdots * \tilde{L}_{p} * \tilde{L}_{1}^{\prime} * \cdots * \tilde{L}_{n}^{\prime}$ be an alternative diagram of $\tilde{L}$. Let $J_{L}(t)$ be the reduced Jones polynomial of $L$. Then the second main theorem of this paper is

THEOREM 9.2.

$$
\begin{aligned}
\text { (1) } \quad l-\operatorname{deg} J_{L}(t) \geq & \sum_{i=1}^{p} g\left(L_{i}\right)-\sum_{j=1}^{n}\left\{h\left(\tilde{L}_{j}^{\prime}\right)+g\left(L_{j}^{\prime}\right)+\mu\left(L_{j}^{\prime}\right)-1\right\} \\
& -\{\mu(L)-1\}+s(L) . \\
\text { (2) } h-\operatorname{deg} J_{L}(t) \leq & \sum_{i=1}^{p}\left\{h\left(\tilde{L}_{i}\right)+g\left(L_{i}\right)\right\} \\
& -\sum_{j=1}^{n}\left\{g\left(L_{j}^{\prime}\right)+\mu\left(L_{j}^{\prime}\right)-1\right\}+s(L) .
\end{aligned}
$$

Therefore, we have

COROLlaRY 9.3. $r-\operatorname{deg} J_{L}(t) \leq h(\tilde{L})+\{\mu(L)-1\}$. If $L$ is split, then

$$
r-\operatorname{deg} J_{L}(t) \leq h(\tilde{L})+\{\mu(L)-1\}+\{\lambda(L)-1\} .
$$

A proof of Theorem 9.2 will be by induction on $h(\tilde{L})$, and the details will be given in the next section. However, since our proof is quite similar to that of Theorem 2.1 , most simple or straightforward calculations will be omitted.

10. Proof of Theorem 9.2. First we deduce a reduction formula similar to (5.5) or (5.6).

Let $c_{1}, \ldots, c_{k}$ be the double points on $\tilde{L}$ to which we apply the fundamental identity (2.1). Let $v$ and $w$ be, respectively, the number of positive and negative double points among these double points $(k=v+w)$. Then the same argument 
used in $\S 5$ gives us the reduction formula

$$
\begin{aligned}
P(L ; l, m)= & (-1)^{k} l^{2(v-w)} P\left(L_{1,2, \ldots, k} ; l, m\right) \\
& +(-1) \sum_{d=1}^{k} \frac{(l m)^{d}}{l^{2 u}} \sum_{1 \leq j_{1}<\cdots<j_{d} \leq k} P\left(L_{j_{1}, \ldots, j_{d}}^{*} ; l, m\right),
\end{aligned}
$$

where $u$ is the number of negative double points among $c_{j_{1}}, \ldots, c_{j_{d}} \cdot(10.1)$ now implies

$$
\begin{aligned}
Q(L ; l, m)= & (-1)^{k} l^{2(v-w)} Q\left(L_{1,2, \ldots, k} ; l, m\right) \\
& +(-1) \sum_{d=1}^{k} \frac{(l m)^{d}}{l^{2 u}}\left(\frac{m}{l}\right)^{\mu-1} \sum_{1 \leq j_{1}<\cdots<j_{d} \leq k} P\left(L_{j_{1}, \ldots, j_{d}}^{*} ; l, m\right),
\end{aligned}
$$

where $\mu=\mu(L)$.

Set $L^{*}=L_{j_{1}, \ldots, j_{d}}^{*}$ and

$$
R\left(j_{1}, \ldots, j_{d}\right)=\frac{(l m)^{d}}{l^{2 u}}\left(\frac{m}{l}\right)^{\mu-1} P\left(L^{*} ; l, m\right) .
$$

Let $R^{*}$ be the polynomial on $t^{ \pm 1}$ which is obtained from $R\left(j_{1}, \ldots, j_{d}\right)$ by the substitutions $l=t \sqrt{-1}$ and $m=-(\sqrt{t}-1 / \sqrt{t}) \sqrt{-1}$. We will prove

PROPOSITION 10.1.

(1) $\quad l-\operatorname{deg} R^{*} \geq \sum_{i=1}^{p} g\left(L_{i}\right)-\sum_{j=1}^{n}\left\{h\left(\tilde{L}_{j}^{\prime}\right)+g\left(L_{j}^{\prime}\right)+\mu\left(L_{j}^{\prime}\right)-1\right\}$

$$
\begin{gathered}
-\{\mu(L)-1\}+s(L) . \\
\text { (2) } h-\operatorname{deg} R^{*} \leq \sum_{i=1}^{p}\left\{h\left(\tilde{L}_{i}\right)+g\left(L_{i}\right)\right\}-\sum_{j=1}^{n}\left\{g\left(L_{j}^{\prime}\right)+\mu\left(L_{j}^{\prime}\right)-1\right\}+s(L) .
\end{gathered}
$$

PROOF. Since a simple calculation shows that

$$
R^{*}=(-1)^{d}\left(t-2+t^{-1}\right)^{d-q} t^{2 q-2 u} J\left(L^{*} ; t\right),
$$

where $2 q=\mu\left(L^{*}\right)-\mu(L)+d$, we have

$$
\begin{aligned}
& \text { (1) } l-\operatorname{deg} R^{*}=3 q-2 u-d+l-\operatorname{deg} J\left(L^{*} ; t\right), \\
& \text { (2) } h-\operatorname{deg} R^{*}=d+q-2 u+h-\operatorname{deg} J\left(L^{*} ; t\right) .
\end{aligned}
$$

Write $L^{*}=L_{1}^{*} * \cdots * L_{p^{*}}^{*} * L_{1}^{\prime *} * \cdots * L_{n^{*}}^{\prime *}$, where $L_{i}^{*}$ are positive links and $L_{j}^{\prime *}$ are negative links. Then the induction assumption on $L^{*}$ gives us

$$
\text { (1) } \begin{aligned}
l-\operatorname{deg} J\left(L^{*} ; t\right) \geq & \sum_{i=1}^{p^{*}} g\left(L_{i}^{*}\right)-\sum_{j=1}^{n^{*}}\left\{h\left(\tilde{L}_{j}^{\prime *}\right)+g\left(L_{j}^{\prime *}\right)+\mu\left(L_{j}^{\prime *}\right)-1\right\} \\
& +s\left(L^{*}\right)-\left\{\mu\left(L^{*}\right)-1\right\}
\end{aligned}
$$

(2) $h-\operatorname{deg} J\left(L^{*} ; t\right) \leq \sum_{i=1}^{p^{*}}\left\{h\left(\tilde{L}_{i}^{*}\right)+g\left(L_{i}^{*}\right)\right\}$

$$
-\sum_{j=1}^{n^{*}}\left\{g\left(L_{j}^{\prime *}\right)+\mu\left(L_{j}^{\prime *}\right)-1\right\}+s\left(L^{*}\right)
$$


where

$$
s\left(L^{*}\right)=\frac{1}{2}\left[\sum_{i=1}^{p^{*}}\left\{\mu\left(L_{i}^{*}\right)-1\right\}+\sum_{j=1}^{n^{*}}\left\{\mu\left(L_{j}^{\prime *}\right)-1\right\}-\left\{\mu\left(L^{*}\right)-1\right\}\right] .
$$

Since $\sum_{i=1}^{p^{*}} h\left(\tilde{L}_{i}^{*}\right)=\sum_{i=1}^{p} h\left(\tilde{L}_{i}\right)-(d-u)$ and $\sum_{j=1}^{n^{*}} h\left(\tilde{L}_{j}^{\prime *}\right)=\sum_{j=1}^{n} h\left(\tilde{L}_{j}^{\prime}\right)-u$, we have

$$
\text { (1) } \sum_{i=1}^{p^{*}} \delta\left(L_{i}^{*}\right)=\sum_{i=1}^{p} \delta\left(L_{i}\right)-(d-u)
$$$$
\text { (2) } \sum_{j=1}^{n^{*}} \delta\left(L_{j}^{\prime *}\right)=\sum_{j=1}^{n} \delta\left(L_{j}\right)-u
$$

Using (4.1)(1) and (10.6), we obtain

$$
\text { (1) } \begin{aligned}
\sum_{i=1}^{p^{*}} g\left(L_{i}^{*}\right)= & \sum_{i=1}^{p} g\left(L_{i}\right)-\frac{1}{2}(d-u)+\frac{1}{2} \sum_{i=1}^{p}\left\{\mu\left(L_{i}\right)-1\right\} \\
& -\frac{1}{2} \sum_{i=1}^{p^{*}}\left\{\mu\left(L_{i}^{*}\right)-1\right\},
\end{aligned}
$$

$$
\text { (2) } \begin{aligned}
\sum_{j=1}^{n^{*}} g\left(L_{j}^{\prime *}\right)= & \sum_{j=1}^{n} g\left(L_{j}^{\prime}\right)-\frac{1}{2} u+\frac{1}{2} \sum_{j=1}^{n}\left\{\mu\left(L_{j}^{\prime}\right)-1\right\} \\
& -\frac{1}{2} \sum_{j=1}^{n^{*}}\left\{\mu\left(L_{j}^{\prime *}\right)-1\right\} .
\end{aligned}
$$

(10.3) is now obtained from (10.4) using (10.5)-(10.7).

Now, to complete the proof of Theorem 9.2 , it only remains to show the following Proposition 10.2. Let $\hat{L}=L_{1,2, \ldots, k}$.

$$
\begin{aligned}
\text { (1) } \quad l-\operatorname{deg} J(\hat{L} ; t)+2(v-w) \geq & \sum_{i=1}^{p} g\left(L_{i}\right)-\sum_{j=1}^{n}\left\{h\left(L_{j}^{\prime}\right)+g\left(L_{j}^{\prime}\right)+\mu\left(L_{j}^{\prime}\right)-1\right\} \\
& -\{\mu(L)-1\}+s(L), \\
\text { (2) } h-\operatorname{deg} J(\hat{L} ; t)+2(v-w) \leq & \sum_{i=1}^{p}\left\{h\left(L_{i}\right)+g\left(L_{i}\right)\right\} \\
& -\sum_{j=1}^{n}\left\{g\left(L_{j}^{\prime}\right)+\mu\left(L_{j}^{\prime}\right)-1\right\}+s(L) .
\end{aligned}
$$

ProOF. Write $\hat{L}=\hat{L}_{1} * \cdots * \hat{L}_{\hat{p}} * \hat{L}_{1}^{\prime} * \cdots * \hat{L}_{\hat{n}}^{\prime}$, where $\hat{L}_{i}$ are positive links and $\hat{L}_{j}^{\prime}$ are negative links. Since $h(\hat{L})=h(\tilde{L})-2 k<h(\tilde{L})$, the induction hypothesis 
yields

(1) $\quad l-\operatorname{deg} J(\hat{L} ; t) \geq \sum_{i=1}^{\hat{p}} g\left(\hat{L}_{i}\right)-\sum_{j=1}^{\hat{n}}\left\{h\left(\hat{L}_{j}^{\prime}\right)+g\left(\hat{L}_{j}^{\prime}\right)+\mu\left(\hat{L}_{j}^{\prime}\right)-1\right\}$

$$
+s(\hat{L})-\{\mu(\hat{L})-1\}
$$

(2) $h-\operatorname{deg} J(\hat{L} ; t) \leq \sum_{i=1}^{\hat{p}}\left\{h\left(\hat{L}_{i}\right)+g\left(\hat{L}_{i}\right)\right\}$

$$
-\sum_{j=1}^{\hat{n}}\left\{g\left(\hat{L}_{j}^{\prime}\right)+\mu\left(\hat{L}_{j}^{\prime}\right)-1\right\}+s(\hat{L})
$$

where

$$
2 s(\hat{L})=\sum_{i=1}^{\hat{p}}\left\{\mu\left(\hat{L}_{i}\right)-1\right\}+\sum_{j=1}^{\hat{n}}\left\{\mu\left(\hat{L}_{j}^{\prime}\right)-1\right\}-\{\mu(\hat{L})-1\} .
$$

From (10.9), we will see that it is enough to show (10.10). Note that $\mu(\hat{L})=\mu(L)$.

$$
\begin{gathered}
\sum_{i=1}^{\hat{p}} g\left(\hat{L}_{i}\right)-\sum_{j=1}^{\hat{n}}\left\{h\left(\hat{L}_{j}^{\prime}\right)+g\left(\hat{L}_{j}^{\prime}\right)+\mu\left(\hat{L}_{j}\right)-1\right\}+s(\hat{L})+2(v-w) \\
\geq \sum_{i=1}^{p} g\left(L_{i}\right)-\sum_{j=1}^{n}\left\{h\left(L_{j}^{\prime}\right)+g\left(L_{j}^{\prime}\right)+\mu\left(L_{j}^{\prime}\right)-1\right\}+s(L),
\end{gathered}
$$

(2) $\sum_{i=1}^{\hat{p}}\left\{h\left(\hat{L}_{i}\right)+g\left(\hat{L}_{i}\right)\right\}-\sum_{j=1}^{\hat{n}}\left\{g\left(\hat{L}_{j}^{\prime}\right)+\mu\left(\hat{L}_{j}^{\prime}\right)-1\right\}+s(\hat{L})+2(v-w)$

$$
\leq \sum_{i=1}^{p}\left\{h\left(L_{i}\right)+g\left(L_{i}\right)\right\}-\sum_{j=1}^{n}\left\{g\left(L_{j}^{\prime}\right)+\mu\left(L_{j}^{\prime}\right)-1\right\}+s(L) .
$$

Since $\sum_{i=1}^{\hat{p}} h\left(\hat{L}_{i}\right)=\sum_{i=1}^{p} h\left(L_{i}\right)-2 v$ and $\sum_{j=1}^{\hat{n}} h\left(\hat{L}_{j}^{\prime}\right)=\sum_{j=1}^{n} h\left(L_{j}^{\prime}\right)-2 w$, using (4.1)(1), (10.10) can be simplified to the following:

$$
\begin{aligned}
& \text { (1) } \frac{1}{2} \sum_{i=1}^{\hat{p}} \delta\left(\hat{L}_{i}\right)-\frac{1}{2} \sum_{j=1}^{\hat{n}} \delta\left(\hat{L}_{j}^{\prime}\right)+2 v \geq \frac{1}{2} \sum_{i=1}^{p} \delta\left(L_{i}\right)-\frac{1}{2} \sum_{j=1}^{n} \delta\left(L_{j}^{\prime}\right), \\
& \text { (2) } \frac{1}{2} \sum_{i=1}^{\hat{p}} \delta\left(\hat{L}_{i}\right)-\frac{1}{2} \sum_{j=1}^{\hat{n}} \delta\left(L_{j}^{\prime}\right)-2 w \leq \frac{1}{2} \sum_{i=1}^{p} \delta\left(L_{i}\right)-\frac{1}{2} \sum_{j=1}^{n} \delta\left(L_{j}^{\prime}\right)
\end{aligned}
$$

Therefore, it is sufficient to show, finally, that

$$
\begin{aligned}
& \text { (1) } \sum_{i=1}^{\hat{p}} \delta\left(\hat{L}_{i}\right)+4 v \geq \sum_{i=1}^{p} \delta\left(L_{i}\right) \geq \sum_{i=1}^{\hat{p}} \delta\left(\hat{L}_{i}\right), \\
& \text { (2) } \sum_{j=1}^{\hat{n}} \delta\left(\hat{L}_{j}^{\prime}\right)+4 w \geq \sum_{j=1}^{n} \delta\left(L_{j}^{\prime}\right) \geq \sum_{j=1}^{\hat{n}} \delta\left(\hat{L}_{j}^{\prime}\right) .
\end{aligned}
$$

Since $\hat{L}_{i}$ and $L_{i}$ are positive links, and $\hat{L}_{j}^{\prime}$ and $L_{j}^{\prime}$ are negative links, (10.11) is exactly the same as (8.9) in Lemma 8.1. 

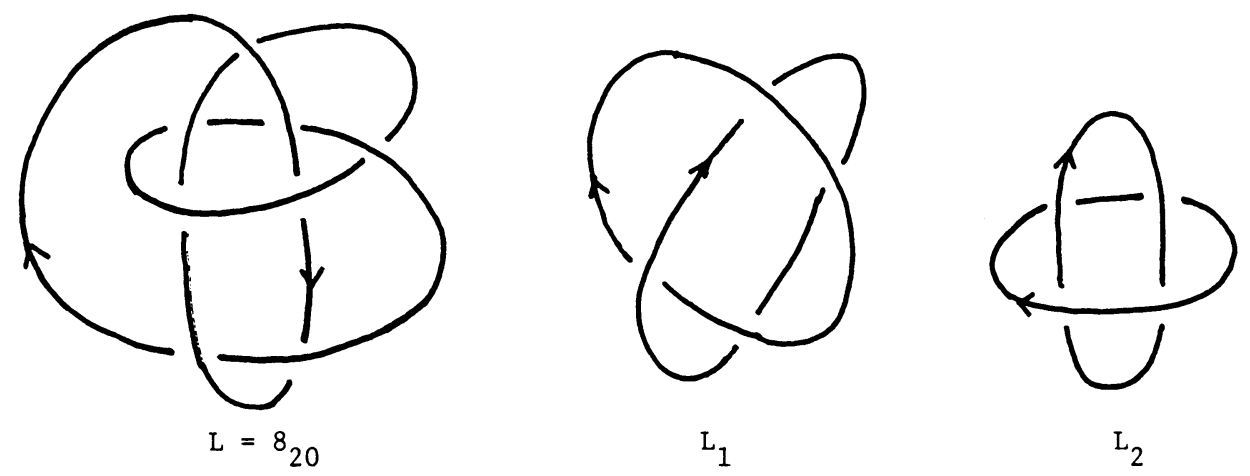

FIGURE 10

The proof of Proposition 10.2 and hence the proof of Theorem 9.2 are now completed.

REMARK. What we have actually proved in Theorem 9.2 is that if $L=L_{1} * \cdots *$ $L_{m}$ is an alternative link, then

$$
r-\operatorname{deg} J(L ; t)-\{\mu(L)-1\} \leq \sum_{i=1}^{m}\left[r-\operatorname{deg} J\left(L_{i} ; t\right)-\left\{\mu\left(L_{i}\right)-1\right\}\right]
$$

This formula is not true for nonalternative links (see Example 10.1). Nevertheless it seems that $r-\operatorname{deg} J(L ; t)-\{\mu(L)-1\}$ is bounded by $h(\tilde{L})$ for any link diagram $\tilde{L}$ of $L$.

EXAMPLE 10.1. A knot $8_{20}$ is the *-product of two links $L_{1}$ and $L_{2}$. (See Figure 10.)

It is known that

$$
\begin{aligned}
& r-\operatorname{deg} J(L ; t)-\{\mu(L)-1\}=6, \\
& r-\operatorname{deg} J\left(L_{1} ; t\right)-\left\{\mu\left(L_{1}\right)-1\right\}=2, \\
& r-\operatorname{deg} J\left(L_{2} ; t\right)-\left\{\mu\left(L_{2}\right)-1\right\}=1 .
\end{aligned}
$$

11. Alternating algebraic links. In this section, we prove that equality in Theorem A holds for alternating algebraic links considered in [13].

Let $T$ be a weighted finite connected tree, and let $w$ be a weight function. Denote by $V(T)$ the set of all vertices in $V$ and by $v(T)$ the number of vertices of $T$ and $w(T)=\sum_{v \in V(T)}|w(v)| . T$ is called even and excessive if for any $v \in V(T), w(v)$ is even and $|w(v)| \geq \max \{\operatorname{val}(v), 2\}$, where $\operatorname{val}(v)$ denotes the valency of $v . v \in V(T)$ is called positive (negative) if $w(v)>0(w(v)<0)$. We assume that $w(v) \neq 0$ for any $v \in V(T)$. A tree is called positive (negative) if all the vertices are positive (negative).

For a weighted tree, let $A(T)$ be the set of those edges in $T$ which join positive vertices and negative vertices. If all the edges in $A(T)$ are removed from $T, T$ will split into finitely many subtrees $T_{1}, \ldots, T_{k}$, each of which is either strictly positive or negative. A collection $\left\{T_{1}, T_{2}, \ldots, T_{k}\right\}$ is called the uniform decomposition of $T$.

Now, given an even connected tree $T$, we can construct an orientable surface $F$ by plumbing as specified by $T$. The boundary of $F$ is an oriented link, denoted by $l(T)$. The orientation of $l(T)$ is induced from that of $F$. 
It is proved in $[\mathbf{1 3}]$.

PROPOSITION 11.1. If $T$ is a positive (negative) even excessive tree, then $l(T)$ is a positive (negative) alternating link. More generally, for an even tree $T$, if each component of the uniform decomposition of $T$ is excessive, then $l(T)$ is an alternating link.

For a proof, see Proposition 4.2 in [13]. The main theorem of this section is

THEOREM 11.2. Let $T$ be an even tree and let $J_{L}(t)$ be the reduced Jones polynomial of the algebraic link $L=l(T)$ associated with $T$. Let $\left\{T_{1}, T_{2}, \ldots, T_{k}\right\}$ be the uniform decomposition of $T$. If each $T_{i}$ is excessive, then

$$
r-\operatorname{deg} J_{L}(t)=w(T)-v(T)+k+\mu(L)-1 .
$$

Furthermore, there is an alternating diagram $\tilde{L}$ of $L$ such that $h(\tilde{L})=w(T)-$ $v(T)+k$. Therefore, $w(T)-v(T)+k$ is the minimum number of double points any alternating diagram of $L$ can have.

Since a 2-bridge knot or link is an algebraic link associated with an even excessive tree, the next corollary follows immediately.

COROLLARY 11.3. Let $L(\alpha, \beta)$ be the 2-bridge link of type $(\alpha, \beta)$, where $\beta$ is odd and $0<\beta<\alpha$. Let $\left\{a_{11}, a_{12}, \ldots, a_{1, n_{1}},-a_{21}, \ldots,-a_{2, n_{2}}, \ldots,(-1)^{k-1} a_{k, 1}, \ldots\right.$, $\left.(-1)^{k-1} a_{k, n_{k}}\right\}$ be a continued fraction form of $\alpha /(\alpha-\beta)$, where $a_{i j}$ are positive and even. Then

$$
r-\operatorname{deg} J_{L}(t)=\sum_{i=1}^{k}\left[\sum_{j=1}^{n_{i}} a_{i, j}-\left(n_{i}-1\right)\right]+\mu(L)-1,
$$

and there is an alternating diagram $\tilde{L}$ of $L$ such that

$$
h(\tilde{L})=\sum_{i=1}^{k}\left[\sum_{j=1}^{n_{i}} a_{i, j}-\left(n_{i}-1\right)\right] .
$$

PROOF OF THEOREM 11.2. We will prove equalities in (9.2) by induction on $v(T)$. If $v(T)=1$, then $k=1$ and $l(T)$ is a torus link of type $(v(T), 2)$. A direct computation then proves Theorem 11.2.

Now we assume that equalities hold in (9.2) for any algebraic link $l\left(T^{\prime}\right)$ with $v\left(T^{\prime}\right)<v(T)$.

Let $\left\{T_{1}, \ldots, T_{k}\right\}$ be the uniform decomposition of $T$. Assume that $T_{1}, \ldots, T_{p}$ are positive (excessive) trees and $T_{p+1}, \ldots, T_{k}$ are negative (excessive) trees. First we note

$$
\begin{aligned}
& \text { For any } i, \\
& \text { (1) } \delta\left(l\left(T_{i}\right)\right)=v\left(T_{i}\right) \quad[\mathbf{1 3}, \text { Proposition 7.1(1)], }
\end{aligned}
$$$$
\text { (2) } h\left(l\left(T_{i}\right)\right)=w\left(T_{i}\right)-v\left(T_{i}\right)+1 \text {. }
$$

Now using (4.1)(1) and (11.3), we compute the right-hand sides of (9.2) and find that it is sufficient to prove the following lemma. 


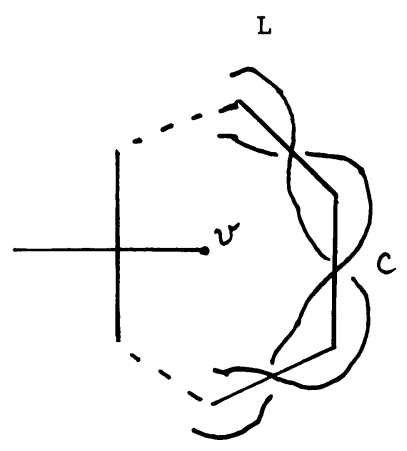

(a)

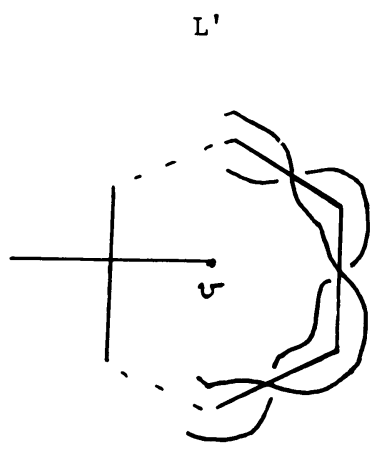

(b)

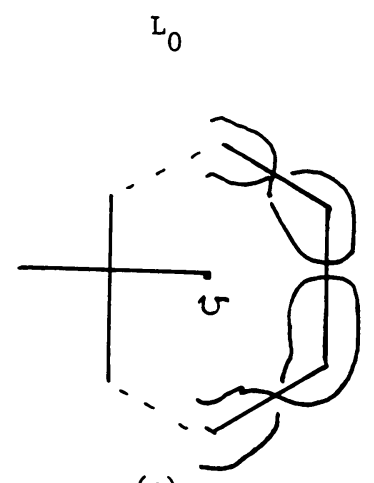

(c)

FIGURE 11

LEMMA 11.4. Let $L=l(T)$. Let $w_{P}(T)=\sum_{i=1}^{p} w\left(T_{i}\right)$ and $w_{N}(T)=$ $\sum_{j=p+1}^{k}\left|w\left(T_{j}\right)\right|$. Then

(1) $\quad l-\operatorname{deg} J(L ; t)=\frac{1}{2} v(T)-w_{N}(T)-\frac{3}{2}\{\mu(L)-1\}-(k-p)$,

(2) $\quad h-\operatorname{deg} J(L ; t)=w_{P}(T)-\frac{1}{2} v(T)-\frac{1}{2}\{\mu(L)-1\}+p$.

Proof. Take a stump $v$; i.e., $\operatorname{val}(v)=1$. We may assume w.l.o.g. that $v \in$ $T_{k}$. Let $w(v)=\nu<0$. Applying the fundamental identity at a double point $c$, illustrated in Figure 11(a), we have

$$
\begin{aligned}
(-1) Q(L ; l, m) & =l^{-2} Q\left(L^{\prime} ; l, m\right)+(m / l)^{\mu} P\left(L_{0} ; l, m\right) \\
& =l^{-2} Q\left(L^{\prime} ; l, m\right)+(m / l)^{\mu-\mu_{0}+1} Q\left(L_{0} ; l, m\right),
\end{aligned}
$$

where $\mu=\mu(L)$ and $\mu_{0}=\mu\left(L_{0}\right)$.

Let $T^{\prime}$ and $T_{0}$ be weighted trees with which algebraic links $L^{\prime}$ and $L_{0}$ are associated.

Case 1. $|w(v)|=|\nu|=2$.

Let $v^{\prime}$ be a vertex of $T_{k}$ incident to $v$. (We assume $v\left(T_{k}\right) \geq 2$. For the case $V\left(T_{k}\right)=\{v\}$, the proof is similar and omitted.) Then $\left\{T_{1}, \ldots, T_{k-1}, \hat{T}_{k}\left(v, v^{\prime}\right)\right\}$ is the uniform decomposition of $T^{\prime}$, where $\hat{T}_{k}(H)$ denotes the complement of a subtree $H$ of $T_{k} .\left(\hat{T}_{k}(H)\right.$ is the tree obtained from $T_{k}$ by removing all vertices of $H$ and all edges of $T_{k}$ incident with at least one vertex in $H . \hat{T}_{k}(H)$ is, in general, a collection of trees.) Furthermore, $\left\{T_{1}, \ldots, T_{k-1}, \hat{T}_{k}(v)\right\}$ is the uniform decomposition of $T_{0}$.

Case 1(i). $\mu-\mu_{0}=1$.

(11.5) yields

$$
(-1) J(L ; t)=(-1) t^{-2} J\left(L^{\prime} ; t\right)+\left(t-2+t^{-1}\right) t^{-2} J\left(L_{0} ; t\right) .
$$

We then prove

$$
\begin{aligned}
& \text { (1) } l-\operatorname{deg} J\left(L^{\prime} ; t\right)-2>l-\operatorname{deg} J\left(L_{0} ; t\right)-3, \\
& \text { (2) } h-\operatorname{deg} J\left(L^{\prime} ; t\right)-2<h-\operatorname{deg} J\left(L_{0} ; t\right)-1 \text {. }
\end{aligned}
$$

Since the proof of (2) is similar to that of (1), we only prove (1). 
Since $T_{k}$ is an even excessive tree, $\hat{T}_{k}\left(v, v^{\prime}\right)$ consists of at most $\left|w\left(v^{\prime}\right)\right| / 2$ connected negative trees, and hence the number, $\xi$, of negative trees in the uniform decomposition of $T^{\prime}$ is at most $(k-p-1)+\left|w\left(v^{\prime}\right)\right| / 2$. Note that $\left|w\left(v^{\prime}\right)\right|$ is even and positive. A simple computation then shows that

$$
\begin{aligned}
l-\operatorname{deg} J\left(L^{\prime} ; t\right)-2 & =\frac{1}{2}\{v(T)-2\}-\left\{w_{N}(T)-2-\left|w\left(v^{\prime}\right)\right|\right\}-\frac{3}{2}\left\{\mu\left(L^{\prime}\right)-1\right\}-\xi-2 \\
& \geq l-\operatorname{deg} J\left(L_{0} ; t\right)-3+\frac{\left|w\left(v^{\prime}\right)\right|}{2}>l-\operatorname{deg} J\left(L_{0} ; t\right)-3 .
\end{aligned}
$$

This proves (11.7).

Now we see from (11.6) that

$$
\begin{aligned}
l-\operatorname{deg} J(L ; t) & =l-\operatorname{deg} J\left(L_{0} ; t\right)-3 \\
& =\frac{1}{2} v(T)-w_{N}(T)-\frac{3}{2}\{\mu(L)-1\}-(k-p) .
\end{aligned}
$$

This proves (11.4)(1). A proof of (2) is omitted.

Case 1(ii). $\mu-\mu_{0}=-1$.

(11.5) yields

$$
(-1) J(L ; t)=(-1) t^{-2} J\left(L^{\prime} ; t\right)+J\left(L_{0} ; t\right) .
$$

Then we can prove

(1) $l-\operatorname{deg} J\left(L^{\prime} ; t\right)-2>l-\operatorname{deg} J\left(L_{0} ; t\right)$,

(2) $h-\operatorname{deg} J\left(L^{\prime} ; t\right)-2<h-\operatorname{deg} J\left(L_{0} ; t\right)$.

Therefore, $l$-deg $J(L ; t)=l-\operatorname{deg} J\left(L_{0} ; t\right)$ and $h-\operatorname{deg} J(L ; t)=h-\operatorname{deg} J\left(L_{0} ; t\right)$, and we obtain (11.4). The details will be omitted.

Case 2. $|w(v)|=|\nu|>2$.

Let $T_{k}^{\prime}$ be the same weighted tree as $T_{k}$ except for one weight at $v$, where $w(v)=$ $-(\nu-2)$. Then $\left\{T_{1}, \ldots, T_{k-1}, T_{k}^{\prime}\right\}$ is the uniform decomposition of $T^{\prime} . T_{0}$ is the same as before. Then we can prove

$$
\begin{aligned}
& \text { (I) If } \mu-\mu_{0}=1 \text {, then } \\
& \text { (1) } l-\operatorname{deg} J\left(L^{\prime} ; t\right)-2<l-\operatorname{deg} J\left(L_{0} ; t\right)-3, \\
& \text { (2) } h-\operatorname{deg} J\left(L^{\prime} ; t\right)-2<h-\operatorname{deg} J\left(L_{0} ; t\right)-1 \text {. } \\
& \text { (II) If } \mu-\mu_{0}=-1, \text { then } \\
& \text { (1) } l-\operatorname{deg} J\left(L^{\prime} ; t\right)-2<l-\operatorname{deg} J\left(L_{0} ; t\right), \\
& \text { (2) } h-\operatorname{deg} J\left(L^{\prime} ; t\right)-2<h-\operatorname{deg} J\left(L_{0} ; t\right) .
\end{aligned}
$$

These formulas imply (11.4). Since the proofs are straightforward computations, they are omitted.

The proof of (11.1) is now completed.

A construction of an alternating minimum diagram $\tilde{L}$ can be found in the proof of Proposition 4.2 in [13]. Q.E.D.

EXAMPLE 11.1. See Figure 12.

For any positive (or negative) excessive (not necessarily even) connected tree $T$, $l(T)$ is an alternating link, and an alternating (nonoriented) diagram $\widetilde{l(T)}$ of $l(T)$ is naturally obtained from $T$. (For details, see $[\mathbf{1 3}]$.) Since $r$ - $\operatorname{deg} J(l(T) ; t)$ does not depend on an orientation of each component of $l(T)$ [6 or 9], $r$-deg $J(l(T) ; t)$ is well defined for each tree $T$. It seems that Theorem 11.2 is true for these links. 

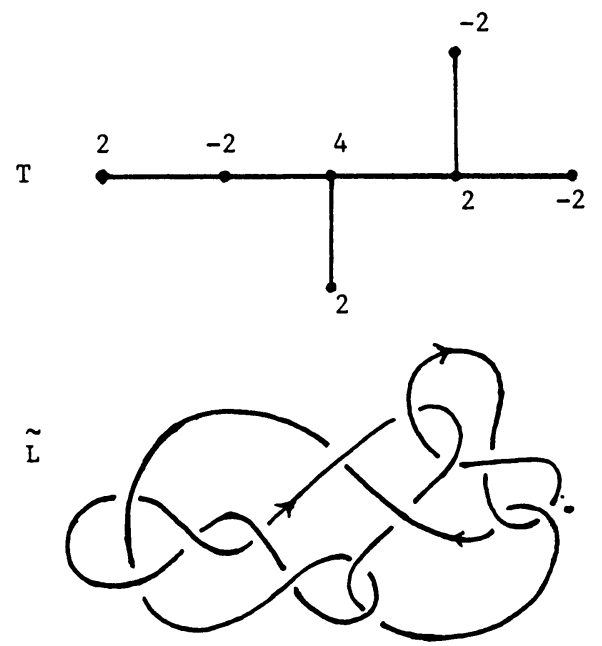

FIGURE 12

Therefore

CONJECTURE. For a positive excessive connected tree $T$,

$$
\begin{gathered}
r-\operatorname{deg} J(l(T) ; t)=w(T)-v(T)+\mu(l(T)), \\
h(l(T))=w(T)-v(T)+1 .
\end{gathered}
$$

Therefore, $w(T)-v(T)+1$ is the minimum number of double points any alternating diagram of $l(T)$ can have.

ADDED IN PROOF. The author proves the above conjecture in the forthcoming paper [14].

\section{REFERENCES}

1. C. Bankwitz, Über die Torsionszahlen der alternierenden Knoten, Math. Ann. 103 (1930), 145-161.

2. R. H. Crowell, Genus of alternating link types, Ann. of Math. (2) 69 (1959), 258-275.

3. _ Non-alternating links, Illinois J. Math. 3 (1959), 101-120.

4. D. Gabai, The Murasugi sum is a natural geometric operation, Contemp. Math. 20 (1983), 131-143.

5. V. Jones, A polynomial invariant for knots via von Neumann algebras, Bull. Amer. Math. Soc. (N.S.) 12 (1985), 103-111.

6. V. F. R. Jones and J. Birman, Seminar notes.

7. L.H. Kauffman, Formal knot theory, Math. Notes, no. 30, Princeton Univ. Press, Princeton, N.J., 1983.

8. W. B. R. Lickorish and K. C. Millett, Polynomial invariant of oriented links (to appear).

9. H. R. Morton, The Jones polynomial for unoriented links (to appear).

10. H. Murakami, A recursive calculation of the Arf invariant of a link (to appear).

11. K. Murasugi, On the genus of the alternating knot. I. II, J. Math. Soc. Japan 10 (1958), 94-105; ibid. 10 (1958), 235-248.

12. __ On a certain numerical invariant of link types, Trans. Amer. Math. Soc. 117 (1965), 387-422.

13. , On the Alexander polynomial of alternating algebraic knots, J. Austral. Math. Soc. (A) 39 (1985), 317-333.

14. __ Jones polynomials and classical conjectures in knot theory (to appear).

Department of Mathematics, University of Toronto, Toronto, Canada, M5S 1A1 